\title{
The Application of Bioinspired Sonar to Cable Tracking on the Seafloor
}

\author{
Keith Brown, Chris Capus, Yan Pailhas, Yvan Petillot, and David Lane \\ School of Engineering and Physical Sciences, Oceans Systems Laboratory, Heriot Watt University, \\ Edinburgh, Scotland EH14 4AS, UK \\ Correspondence should be addressed to Keith Brown, k.e.brown@hw.ac.uk
}

Received 15 June 2010; Accepted 9 September 2010

Academic Editor: Elias Aboutanios

Copyright ( $\odot 2011$ Keith Brown et al. This is an open access article distributed under the Creative Commons Attribution License, which permits unrestricted use, distribution, and reproduction in any medium, provided the original work is properly cited.

Marine mammals have developed highly effective sonar systems for detecting, identifying, and following underwater objects. In this paper we demonstrate that bio-inspired wideband sonar offers great capability for tracking cables on the seafloor. The analysis of biological signals, including dolphin clicks, suggests two approaches. The first is to use a wideband signal, integrating the response of an object over many frequencies. For simple forms, this is known to give access to shape and material information. The second idea is to use intelligent signals designed to elicit information from specific target types. In this paper results are presented from sets of experiments using bio-inspired wideband sonar. The aim of these experiments is to determine the feasibility of tracking small diameter marine communications cables using the wideband responses. Echoes from four different cable types are analysed using a variety of signals. Experiments using bio-inspired pulses illustrate the benefits of using this type of wideband signal for detection and recognition. A strong correspondence between theoretical and experimental echoes is shown.

\section{Introduction}

The highly effective sonar developed by animals [1-4] has inspired work to improve the performance of man-made sonar systems. In particular, the excellent performance of cetaceans in target detection and recognition has been intensively studied with increasing interest in recent years in the development of biomimetic sonar systems [5, 6]. Dolphins are able to detect objects below the surface as well as those lying on the seafloor surface. The Ocean Systems Laboratory (OSL) is developing a bioinspired wideband acoustic sensing system for autonomous underwater vehicles (AUVs) for improved detection and recognition of subsea objects $[7,8]$. One application for the new system is the autonomous tracking of underwater cables. Autonomous tracking is important as it will permit tracking of many kilometres of cable in the time it would take to follow a few hundred metres of cable with a remotely operated vehicle (ROV). Tracking cables on the seafloor is a difficult but important task. Other work on tracking cables on the seafloor has been published but this has been looking at detecting cable-like objects in sonar imagery rather than looking at the signal returns themselves [9]. Global communications and power distribution networks are dependent on marine cables, with an ever-increasing requirement for fast, accurate and reliable survey. These cables need to be accurately tracked both for integrity management and finding cable breaks. The cables may be on the seafloor or buried and it is important to accurately track them as movements in the cable can indicate the possibility of future failure. Additionally the cables may cross other cables or seafloor structures and it is important to be able to identify specific cables so the right cable is tracked after a crossing point. The bioinspired approach offers potential for cable recognition as well as improved detection, making tracking more robust in cluttered environments.

The pulses used for detection must be robust to noise, and reverberation noise in particular. Wideband dolphinlike signals are expected to provide improvement over narrowband signals through pulse compression. For certain difficult maritime targets, they have also been shown to provide improved detection ranges [10]. Within detection range, research has shown that dolphins can distinguish objects as functions of their shapes, materials or contents with some learning $[1,11]$. So even if the interpretation and 


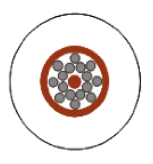

(a)

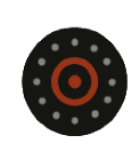

(b)

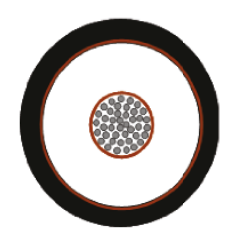

(c)

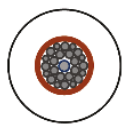

(d)
Plastics

Copper conductor
○ $\bigcirc$ Steel strength members

Fibre optic core
Figure 1: Cross-sections of the four cables used in the experiments.

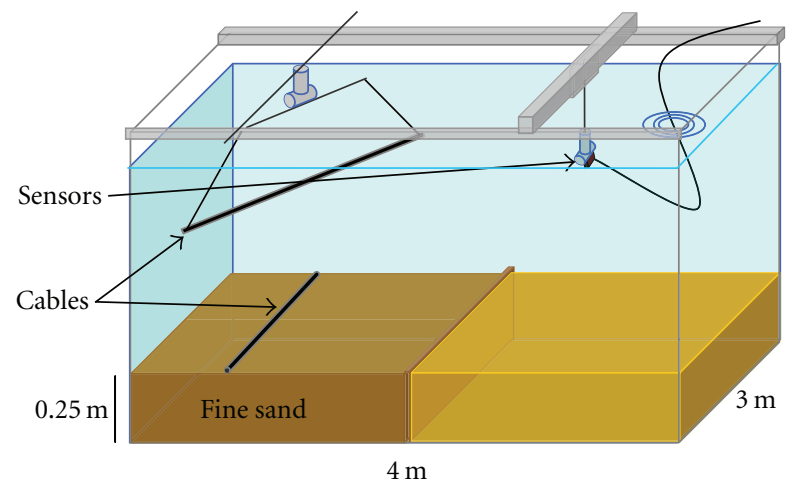

Figure 2: Test tank setup. Cable responses can be measured in free water or against a sediment background. A pan-and-tilt unit is used to rotate the cable section for multiaspect experiments.

identification require complex analysis, this shows that the information hidden in the signal is related to parameters of the target useful for classification purposes.

The purpose of this paper is to describe and analyse the echoes coming from synthetic bioinspired clicks used to track cables lying on and just beneath the seafloor. This paper presents findings from experiments that have been undertaken to determine the feasibility of the bioinspired wideband acoustic approach for marine cable detection and tracking. Cables have been tested for differential response for identification purposes and for discrimination from clutter objects. Multiaspect responses have been measured to determine limits on detection using existing sensors. Limited experimentation has been undertaken with buried cables to determine whether tracking will be possible when cables disappear beneath the surface in soft sediment.

\section{Cables}

Four cable types were used for the experimental programme, these are shown in Figure 1. The external diameters of the first three cables A, B, and C as shown in Figure 1 are $21 \mathrm{~mm}, 16.5 \mathrm{~mm}$, and $32 \mathrm{~mm}$ respectively. Each has two copper conducting elements and a number of steel strands for strength. Cable $\mathrm{C}$, the larger $32 \mathrm{~mm}$ diameter cable, has been used for the majority of the experiments, the other two cables $\mathrm{A}$ and $\mathrm{B}$ have been tested midwater only to

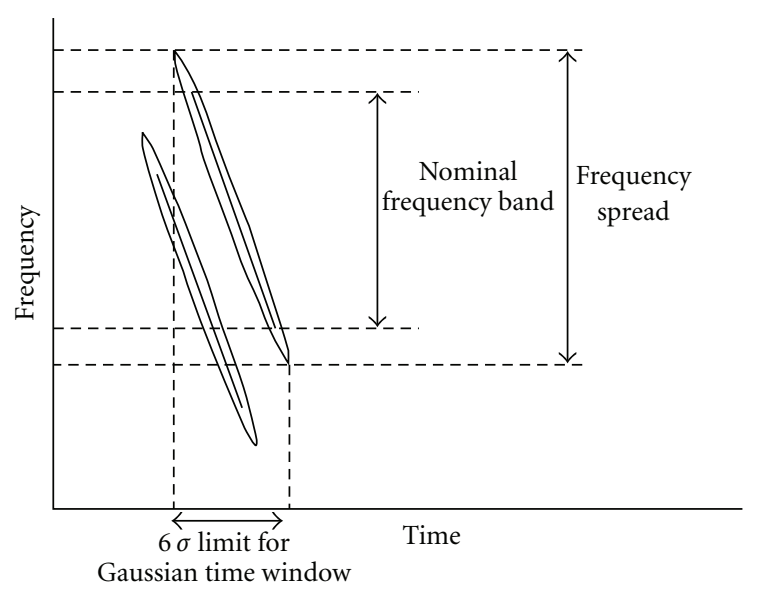

FIGURE 3: Schematic of the time-frequency structure of the DC series double chirp pulses.

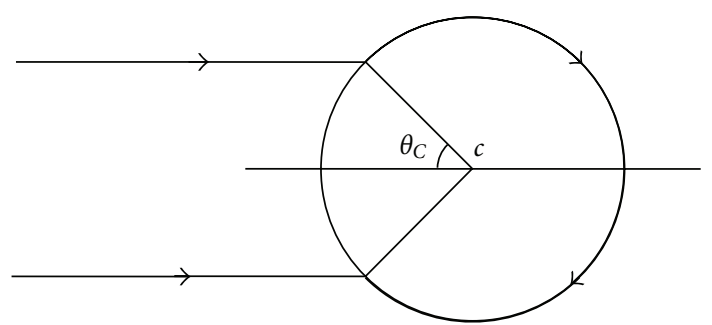

FIgURE 4: Diagram to show Lamb wave propagation path in cylindrical cross-section.

determine baseline wideband responses and potential for discrimination between cable types. Cable D is a section of SL17L a fibre optic cable has also been used for the experiments. This has a similar structure to the type A cable, but is narrower at $17 \mathrm{~mm}$ external diameter. The outer plastic jacket is in medium density polyethylene (MDPE) containing an $8 \mathrm{~mm}$ diameter copper sheathed core. The core comprises 24 steel strength members in three different gauges between $1.13 \mathrm{~mm}$ and $1.59 \mathrm{~mm}$ diameter. The central polybutylene terephthalate (PBT) tube contains a number of optical fibres. This structure bears many similarities with the type A cable. A diagrammatic cross-section for the SL17L cable D is also shown in Figure 1.

The exact compositions of the jacket and dielectric materials used in the cables constructions are not known. Likely candidates are polyurethane, polyethylene, polyvinyl chloride (PVC) and glass reinforced epoxy (GRE). A generic PVC plastic has been assumed for the echo response models reported below.

\section{Experimental Setup}

The basic cable response experimental set up is illustrated in Figure 2. Responses can be measured midwater or against a sediment background, fine sand in this case. For the midwater experiments, cable aspect can be varied in the horizontal plane using a Bowtech PT-25 pan-and-tilt unit. 


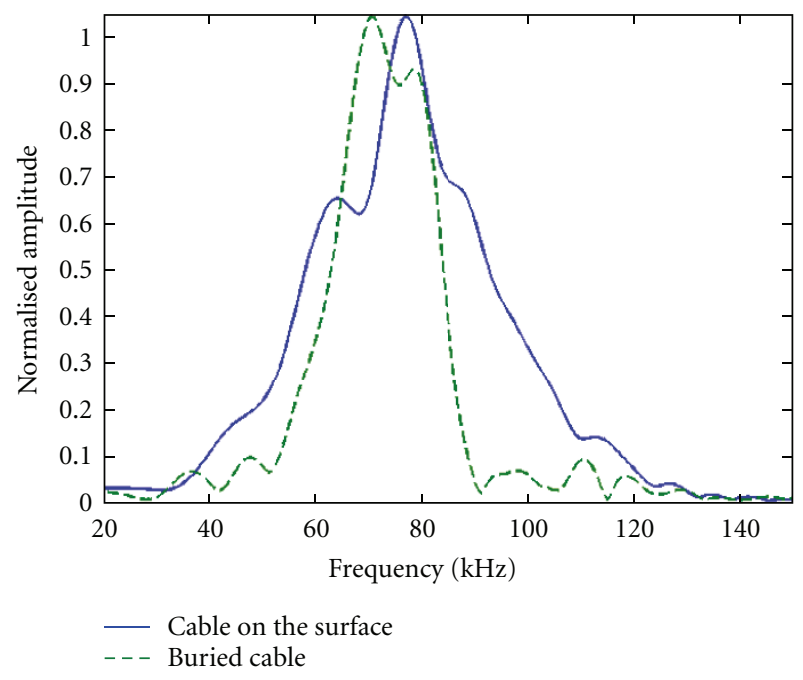

Figure 5: Normalized spectra for the surface cable and for the cable buried with its top surface flush with the sediment.

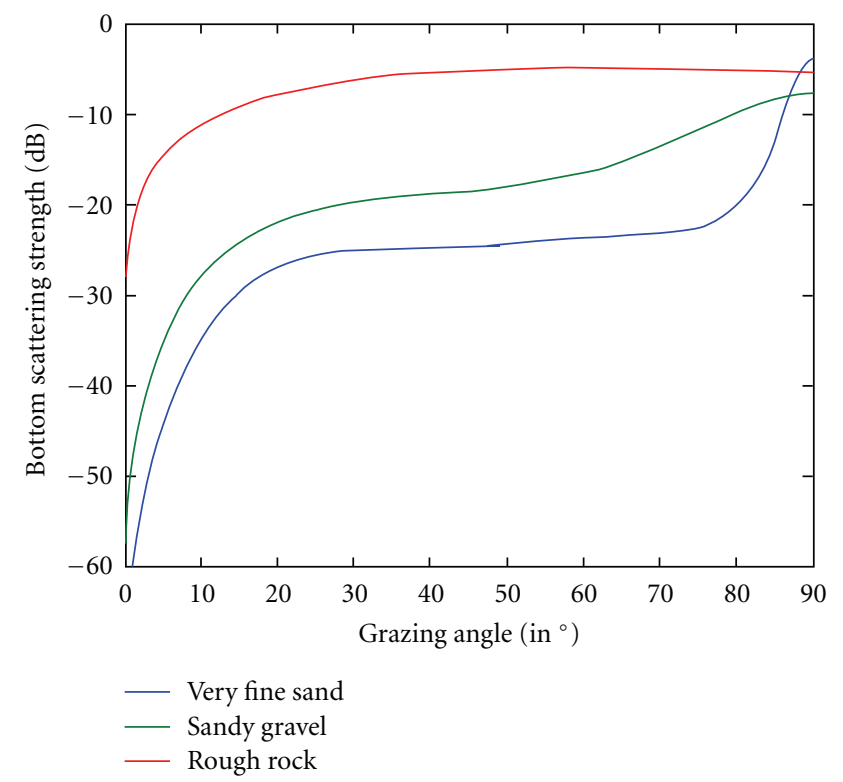

Figure 6: Bottom scattering strengths against grazing angle for three seabed types.

Wideband high and low frequency projectors have been tested with a matched pair of receivers mounted on a PT10 pan-and-tilt unit. The sensor configuration used in these experiments is suitable for vehicle mounting for operation in conjunction with a sidescan sonar and could be readily adapted to run alongside video or other sensors.

3.1. Sensors. The projectors under test each cover around two octaves. The higher frequency unit has a peak response around $100 \mathrm{kHz}$ and $-3 \mathrm{~dB}$ band centre at around $100 \mathrm{kHz}$. The lower frequency projector has a similar level peak response at approximately $85 \mathrm{kHz}$ with the $-3 \mathrm{~dB}$ band centred around $65 \mathrm{kHz}$. In conjunction these sensors are

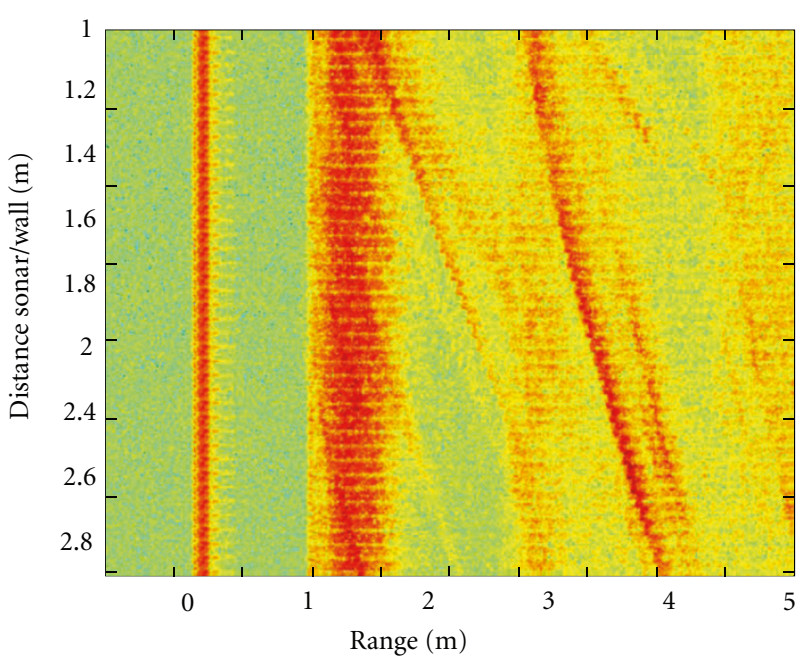

(a)

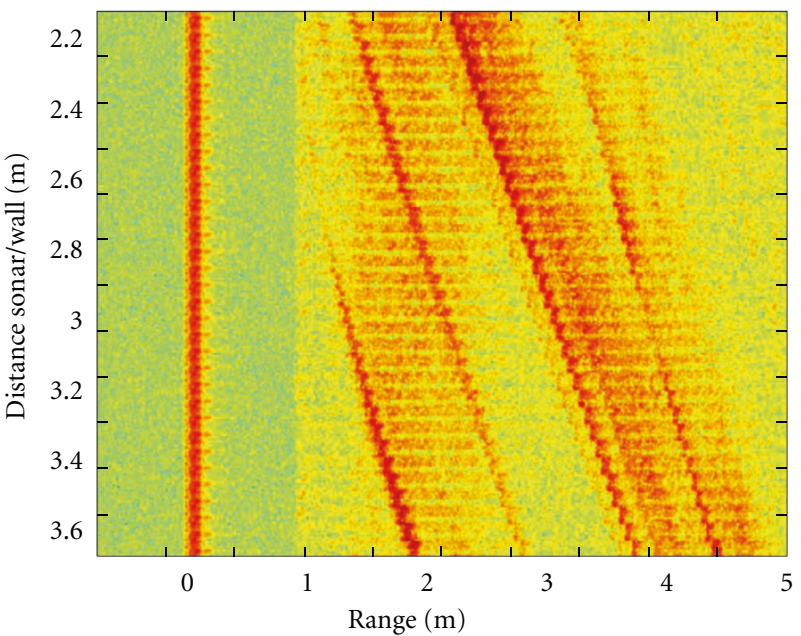

(b)

Figure 7: Series of echo responses from cable lying on sediment as sensor is tracked across the tank: (a) $45^{\circ}$ grazing angle; (b) $27^{\circ}$ grazing angle all six DC pulses recorded at each sensor location.

capable of emitting significant energy in a band ranging from around $30 \mathrm{kHz}$ to $130 \mathrm{kHz}$. The sensors were designed with a prerequisite $-3 \mathrm{~dB}$ bandwidth of $20^{\circ}$ at $120 \mathrm{kHz}$ and $40^{\circ}$ at $60 \mathrm{kHz}$. The cable cross-sections present relatively small targets and in most tests the high-frequency projector has proved most capable. The lower frequency unit may still find application in situations where cable burial is a particular problem.

3.2. Signals. The signals used in this study were developed from previous work analysing bottlenose dolphin signals [7, 10]. These signals comprise a set of six double downchirps. Each pulse covers a nominal bandwidth of $30-130 \mathrm{kHz}$ and comprises two linear downchirp signals each of $100 \mu \mathrm{s}$ duration. These chirp components are constrained by a Gaussian window and between signals only the chirp rate is varied. Frequency spacing between components is set 


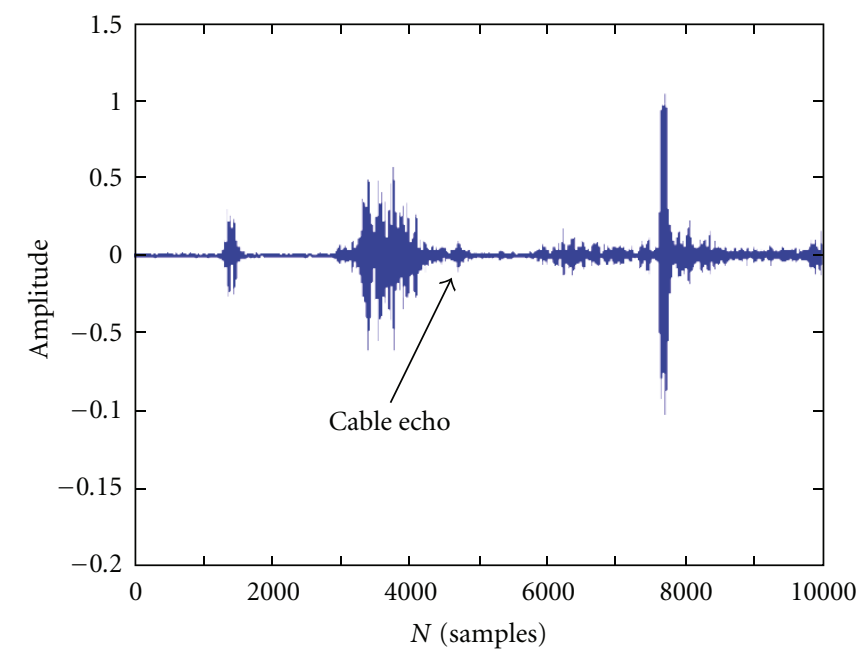

FIGURE 8: Record of DC4 pulse with $45^{\circ}$ grazing angle over very coarse sand-cable echo is arrowed.

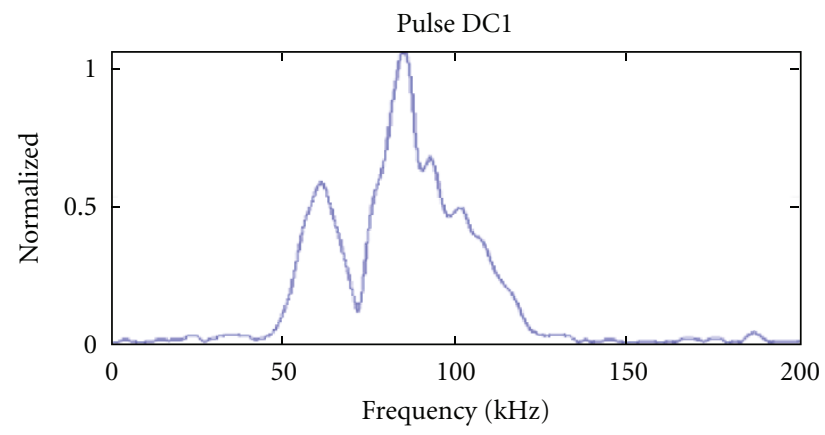

(a)

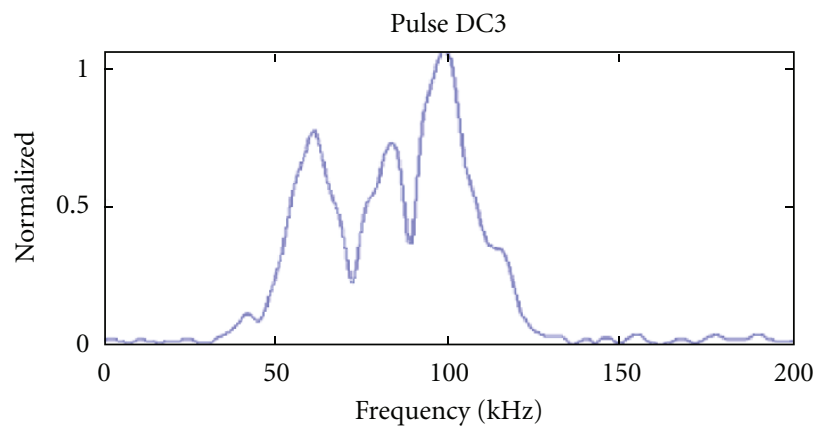

(c)

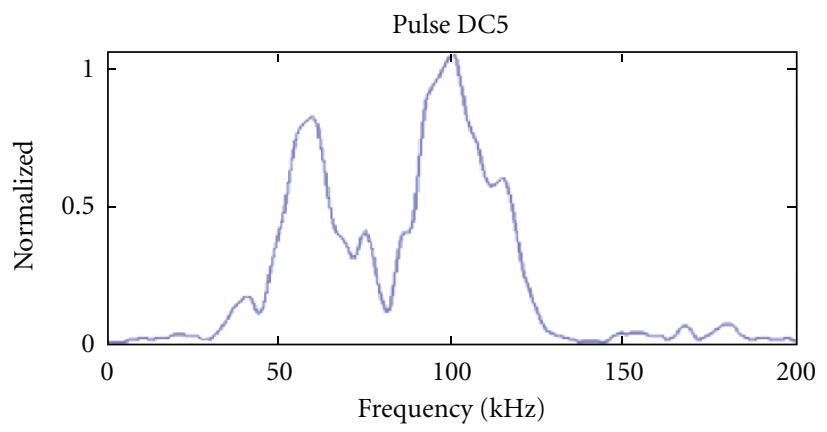

(e)

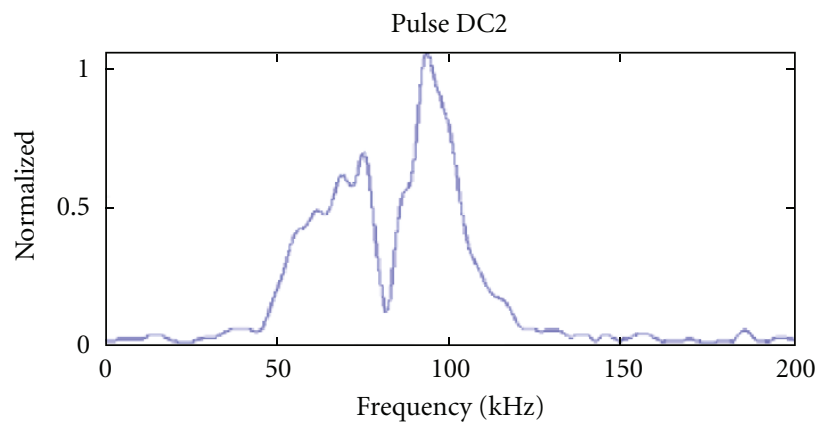

(b)

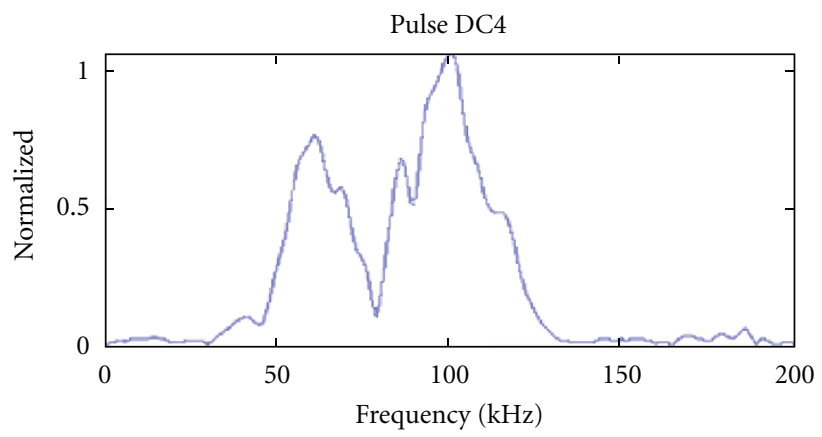

(d)

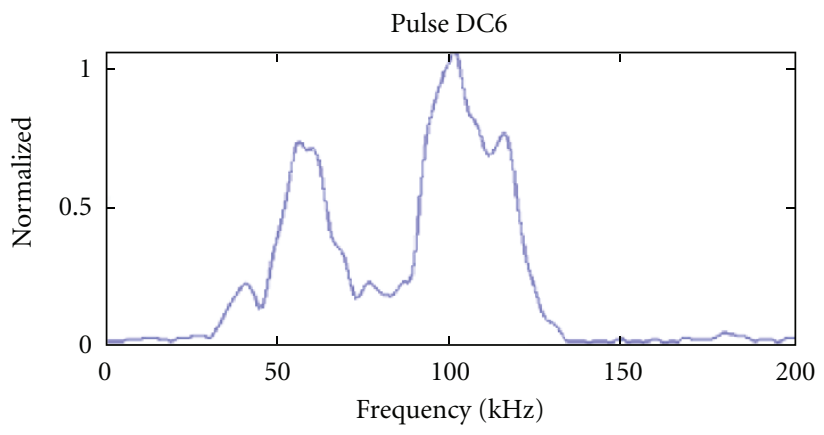

(f)

FIGURE 9: Spectral responses for SL17L cable D on coarse sand, $45^{\circ}$ grazing angle, DC1-6. 


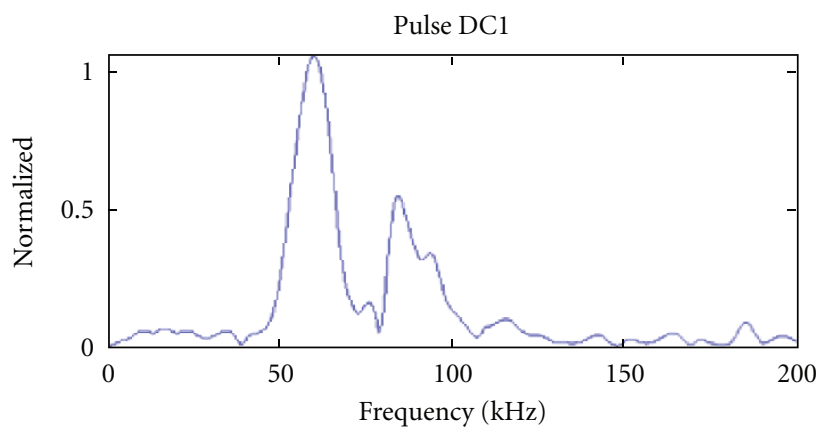

(a)

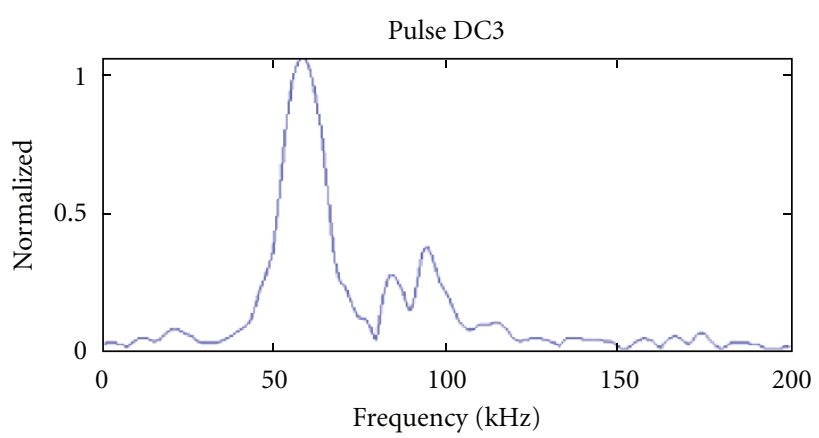

(c)

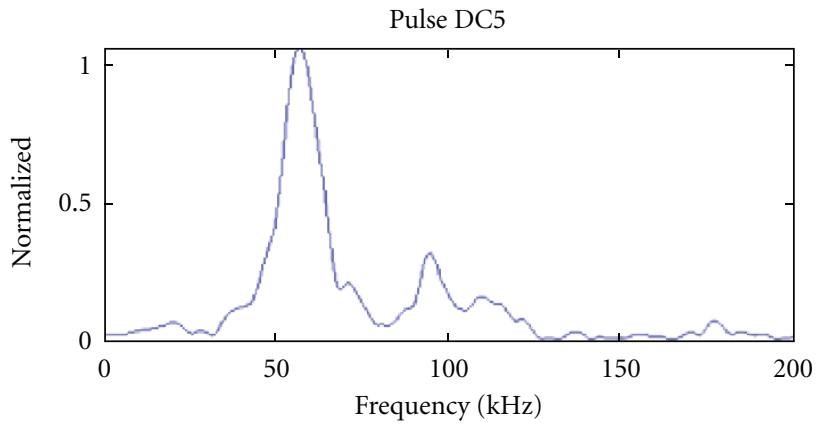

(e)

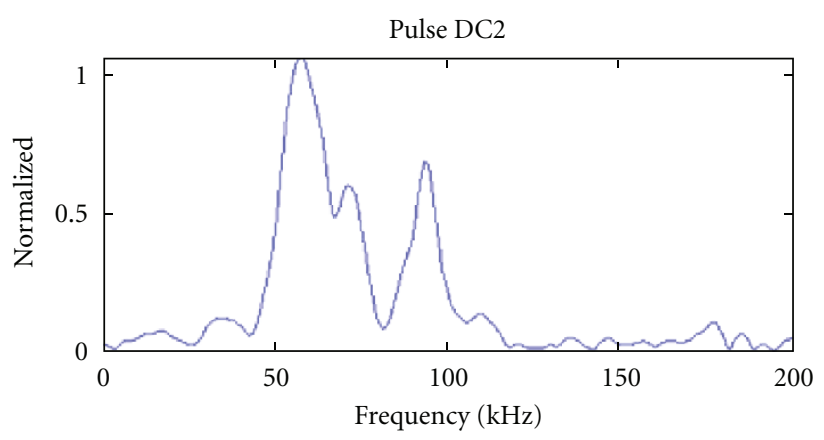

(b)

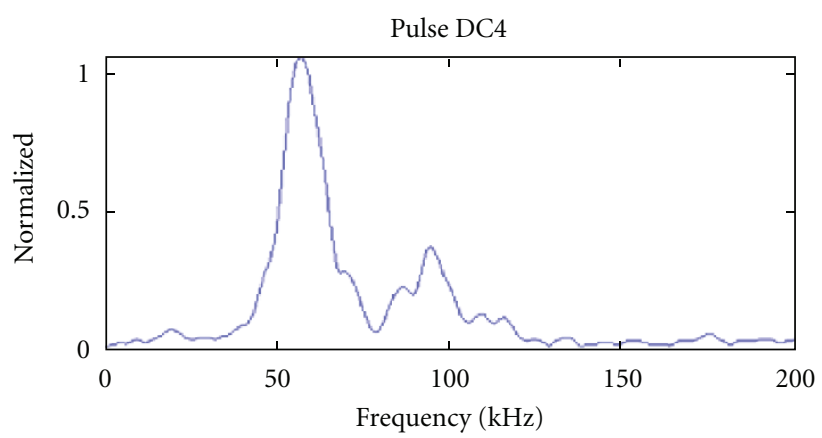

(d)

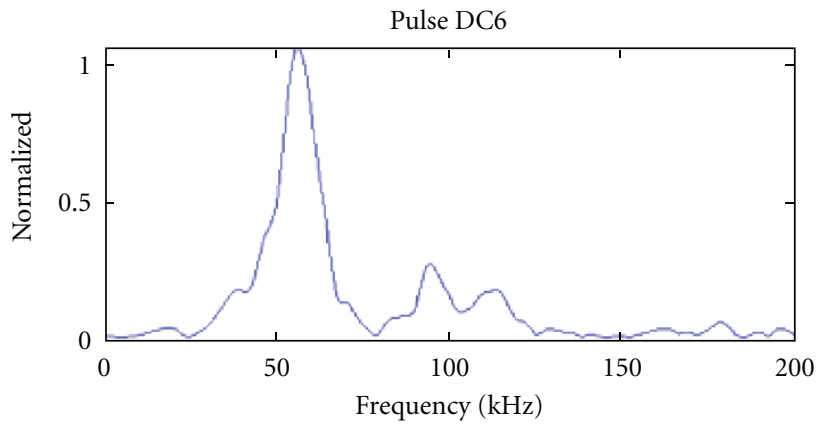

(f)

FIgURE 10: Spectral responses for SL17L cable D on coarse sand, $27^{\circ}$ grazing angle, DC1-6.

to provide the $30-130 \mathrm{kHz}$ range. The higher frequency component is delayed by $20 \mu$ s to produce a total duration of $120 \mu$ s. The pulses labelled DC1 through DC6 have differing degrees of frequency overlap between components, greatest in DC1, which has the highest chirp rates, see Figure 3. In parallel, similar analyses of echolocation pulses used by the big brown bat (Eptesicus fuscus) have indicated that these signals are made up from three or four distinct downchirp components [12]. In related studies on target identification it has been found that these pulses highlight different aspects of a targets wideband response and are most effective used in combination $[8,13]$. Using a general set of pulses in this way reduces the need to tune transmit pulses for specific targets.

\section{Cable Models}

A first cable model has been developed for the type $\mathrm{C}$ cable, which has an external diameter of approximately $32 \mathrm{~mm}$ with
TABLE 1: Thickness parameters for the cable components.

\begin{tabular}{lc}
\hline Component & Thickness \\
\hline Exterior plastic layer & $2.75 \mathrm{~mm}$ \\
Copper layer & $0.3 \mathrm{~mm}$ \\
Inner plastic layer & $8.3 \mathrm{~mm}$ \\
Copper layer & $0.3 \mathrm{~mm}$ \\
Inner steel core (stranded) & $8.7 \mathrm{~mm}$ \\
\hline
\end{tabular}

deformations causing variations of around $1 \mathrm{~mm}$ along its length. Values for the thicknesses of each of the layers, as modelled, are given in Table 1.

Since the physical and acoustic properties of the cable materials are not known precisely, longitudinal and shear velocities for the two plastic layers have been estimated based on a PVC model. This enables us to reach a first approximation for the major backscatter components when the cable 


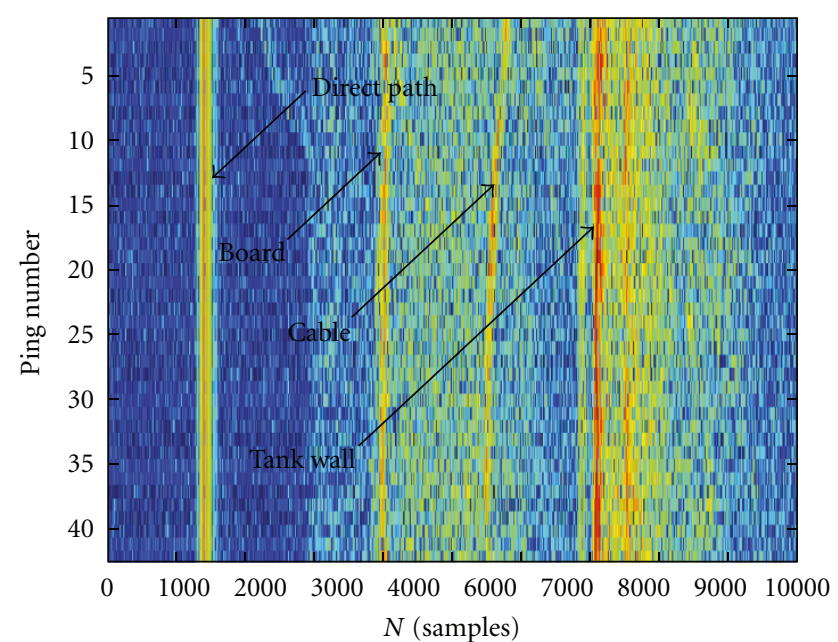

Figure 11: DC3 echoes at $5 \mathrm{~cm}$ intervals along $2 \mathrm{~m}$ section of SL17L cable $\mathrm{D}$ (at $27^{\circ}$ grazing angle).

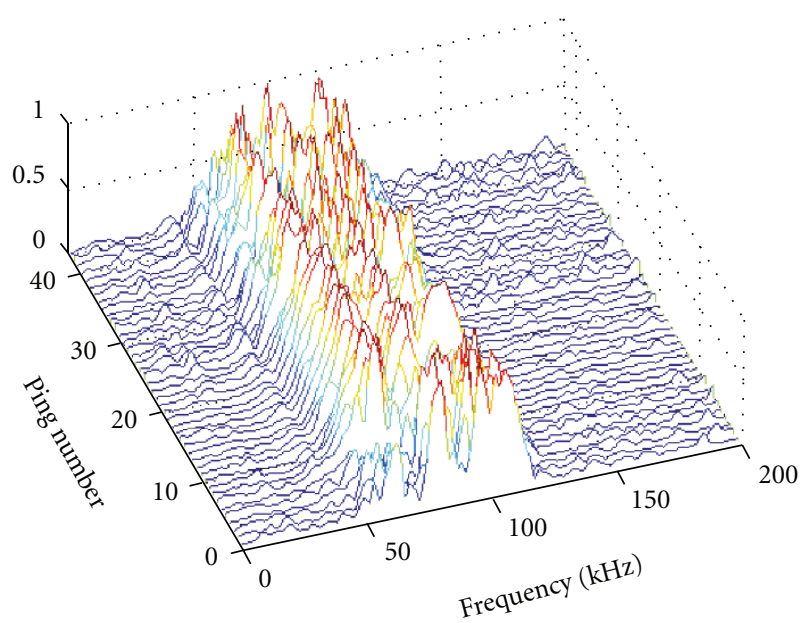

FIGURE 12: Waterfall plot of the cable echo spectra whilst the general three-lobed pattern distinctive for this cable/pulse combination is strongly in evidence, variations would make discrimination from a single ping highly difficult and a more reliable detector will be built by integrating over sequences of returns.

is ensonified by a wideband pulse. Assuming relatively low impedance for the outer plastic sheath, considerable energy will pass through to the relatively high impedance copper layer. The major contributions to the response are formed by the specular echo from the front face, followed by reemanations of surface acoustic waves travelling around the copper conductor. The relative timings of these principal echo components are used to predict the locations of key notch features arising from interferences in the wideband echo spectrum.

The echo response models for the multilayer cable come from the theoretical study of cylindrical shells. The models give the form of the power spectrum of the echo. Two models are presented here. The first, a relatively simple model, helps to explain the structure of the echo power spectrum by

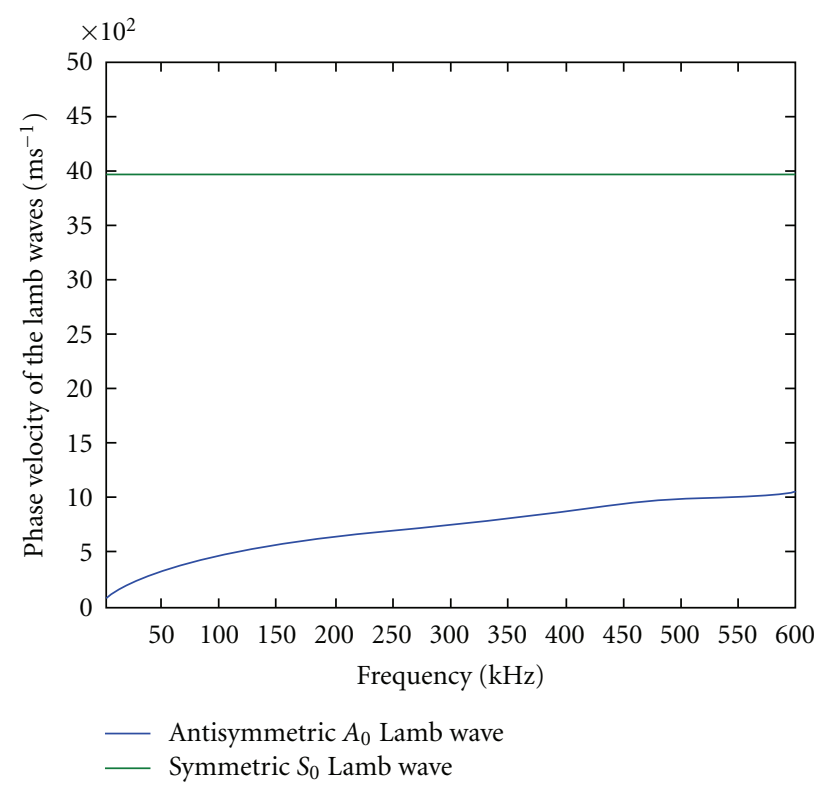

FIGURE 13: Lamb wave phase velocities calculated for the outermost copper layer in the type C cable. Symmetric and antisymmetric waves are shown.

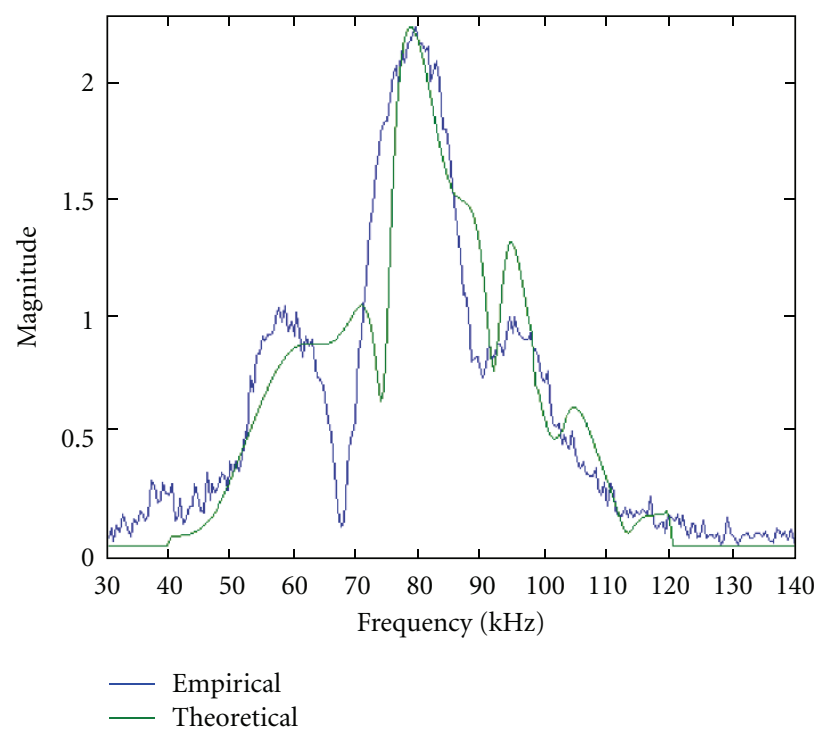

Figure 14: Comparison between theoretical and measured responses for the type $\mathrm{C}$ cable. Spacings between principal notches are well represented. Notch positions are sensitive to relative phases of contributing echoes and are readily affected by any imprecision in values for acoustic properties of the materials.

considering acoustic propagation in a thin plate. The second model solves the wave equation for the layered structure and gives an exact analytical solution.

4.1. Simple Model. In the thin cylindrical shell theory, two models have been proposed depending on the acoustical impedance of the material of the cylinder. Plastic has relatively low impedance (close to that of the surrounding 


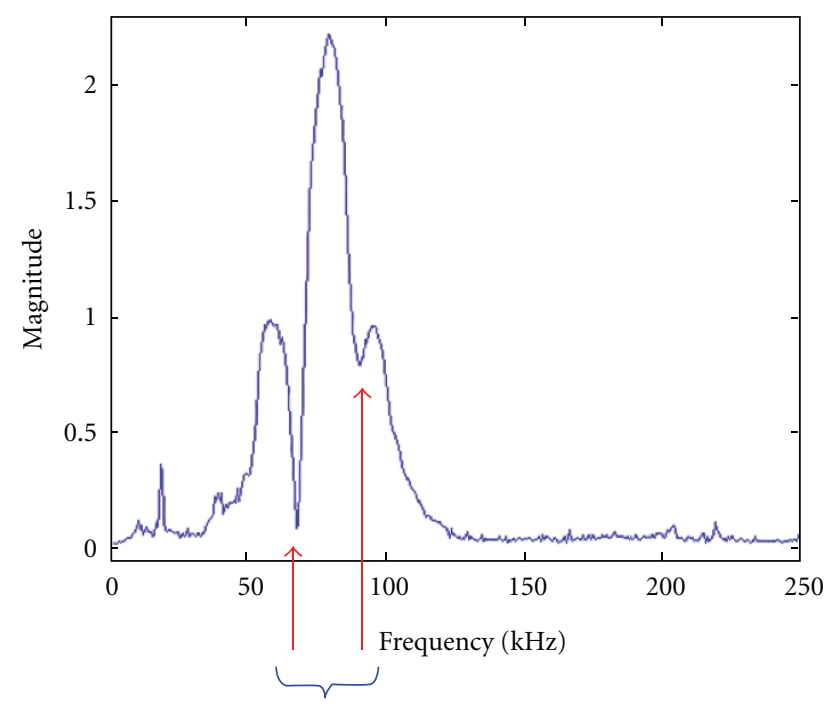

(a)

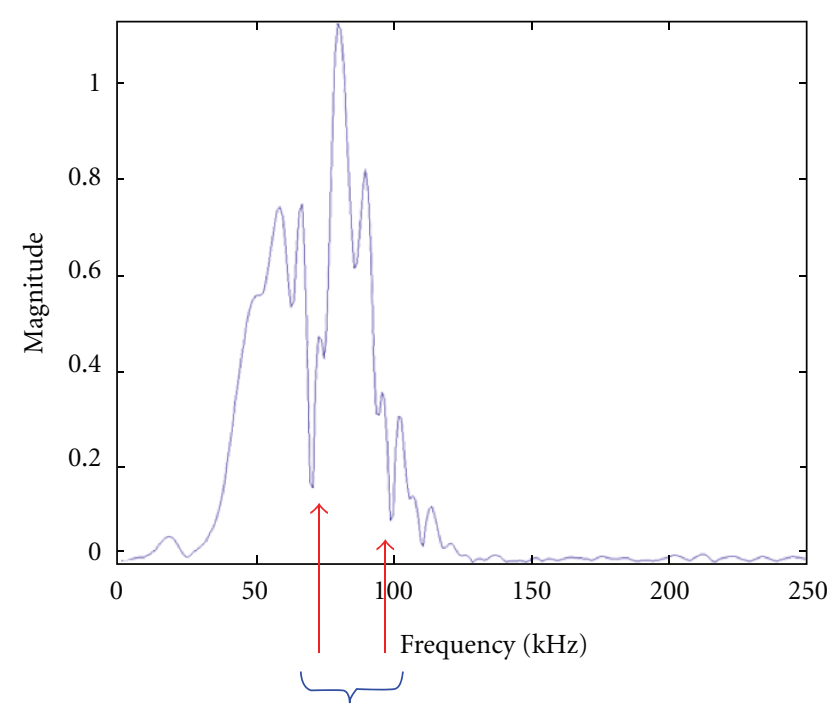

(b)

FIGURE 15: Cable responses: (a) free water, linear FM pulse; (b) DC pulse, cable lying on tank floor. Red arrows indicate model predictions for locations of key spectral responses.

water). This layer will reflect some of the sound, but a large amount of energy is transmitted through the material. In this case the trajectory of the sound can be deduced by a geometrical analogy [8]. Conversely, copper has a high impedance (in comparison with water). The energy transmitted into the interior is very low. However sound does enter into the copper layer itself at the critical angle $\theta_{c}$. It is then propagated around the shell and is back diffracted at the same angle $\theta_{c}$. These phenomena, known as Lamb waves [14-17] and first described for propagation in thin metal plates, are illustrated in Figure 4.

In the case of the cable considered here, both models apply. The first echo or specular echo is the simple reflection at the cable-water interface. Because the first layer is plastic much of the energy is transmitted to the next layer. The secondary echoes are formed predominantly from the reemanation of the sound travelling in the copper layer.

Resolution of the wave equation for a thin plate allows us to compute the sound speed of the Lamb waves. Figure 13 shows the phase velocities in a $0.3 \mathrm{~mm}$ thick copper plate. Typically two kinds of Lamb waves appear: the symmetrical, $S_{0}$, and the anti-symmetrical, $A_{0}$. Because the $A_{0}$ Lamb wave is subsonic (lower than the sound speed in the surrounding water), it does not exist at the frequencies considered. The phase velocity of the $S_{0}$ Lamb wave is constant throughout this frequency band. So, the propagation in this layer is non-dispersive and the group velocity is equal to the phase velocity. The delay between the specular echo and the secondary echoes due to the Lamb wave propagation can be calculated

$$
\Delta t_{n}=2 r\left(\frac{n \pi-\theta_{c}}{v_{g}}-\frac{1-\cos \theta_{c}}{c}\right) .
$$

Note that $n$ represents the number of turns around the cylinder and $r$ is the radius of the copper layer. Numerically we get $\Delta t_{1}=21 \mu \mathrm{s}$ and $\Delta t_{2}=43 \mu \mathrm{s}$. Regular notches seen in the echo spectrum correspond to interferences between the specular and the secondary echoes. Empirically we measure a spacing between the notches of around $23 \mathrm{kHz}$ which corresponds to a time delay of $43.8 \mu$ s. This matches well with the $\Delta t_{2}$ value computed above.

4.2. Analytical Solution of the Wave Equation. Attention is now focused on resolution of the scattering of a cylindrical multilayer structure. We assume that the cylinder is infinitely long. Because of the axial symmetry, the problem is solved in cylindrical coordinates. Assuming the incoming wave is perpendicular to the cylinder, broadside incidence, the problem becomes a 2D problem [17-20]. Each layer is entirely determined by its inner and outer diameter, and the acoustical parameters: density, longitudinal sound speed $c_{l}^{n}$ and shear velocity $c_{t}^{n}$.

Now, let $k_{l}^{n}=2 \pi f / c_{l}^{n}$ and $k_{t}^{n}=2 \pi f / c_{t}^{n}$ be the longitudinal and transversal wave numbers of the different layers $n$. Under these assumptions, the wave equation can be written as follows. In the fluid surrounding the cylinder:

$$
p=p_{i}+p_{s}
$$

where $p$ is the total pressure, $p_{i}$ the incoming pressure and $p_{s}$ is the backscattered pressure. Thanks to the cylindrical symmetry, $p_{i}$ and $p_{s}$ can be written as:

$$
\begin{gathered}
p_{i}=P_{0} \sum_{m=0}^{+\infty} i^{m} \epsilon_{m} J_{m}\left(k_{1} r\right) \cos m \theta, \\
p_{s}=P_{0} \sum_{m=0}^{+\infty} i^{m} \epsilon_{m} b_{m} H_{m}^{(1)}\left(k_{1} r\right) \sin m \theta,
\end{gathered}
$$

where $\epsilon_{n}$ is Neumann factor $\left(\epsilon_{0}=1, \epsilon_{n}=2\right.$ for $\left.n>0\right), P_{0}$ is the pressure amplitude of the incident (incoming) wave, $J_{m}$ is a Bessel function of the first order and $H_{m}^{(1)}$ is a Hankel function of the first order. 

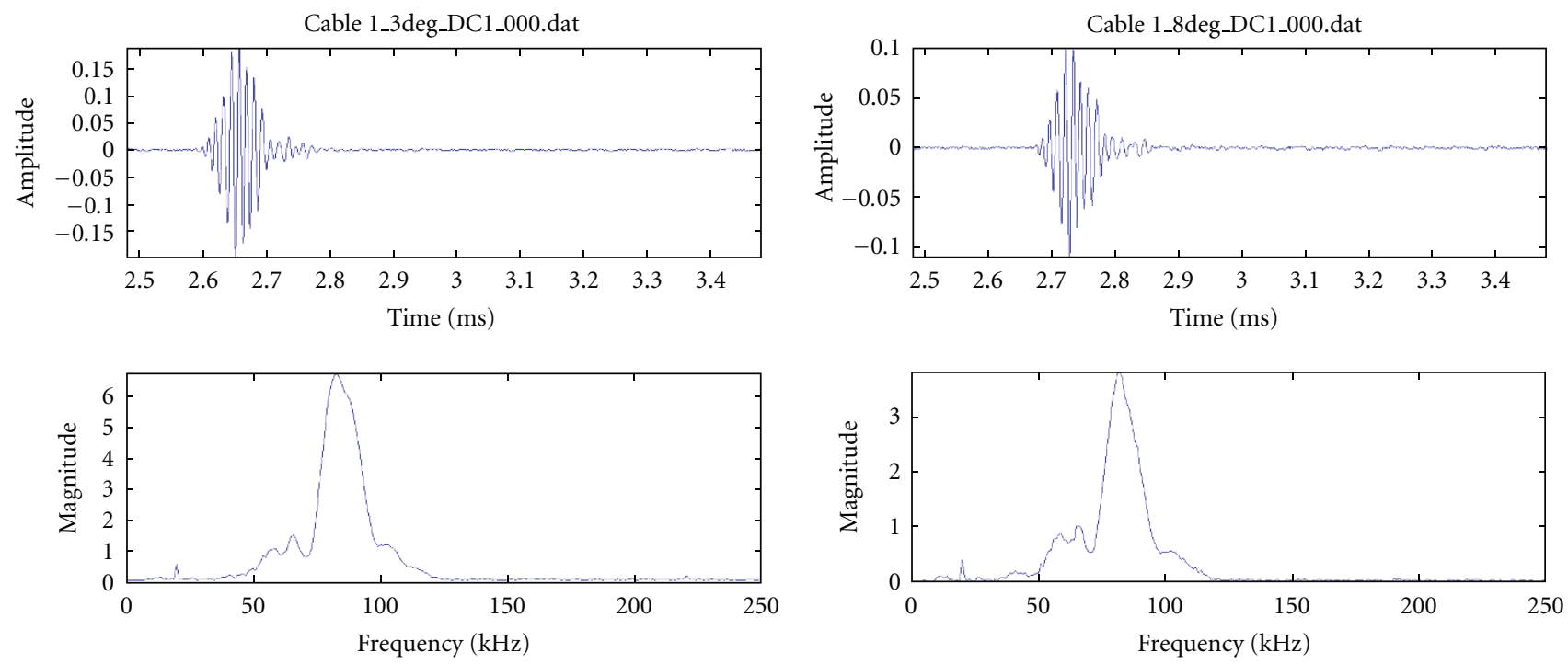

(a)

(b)
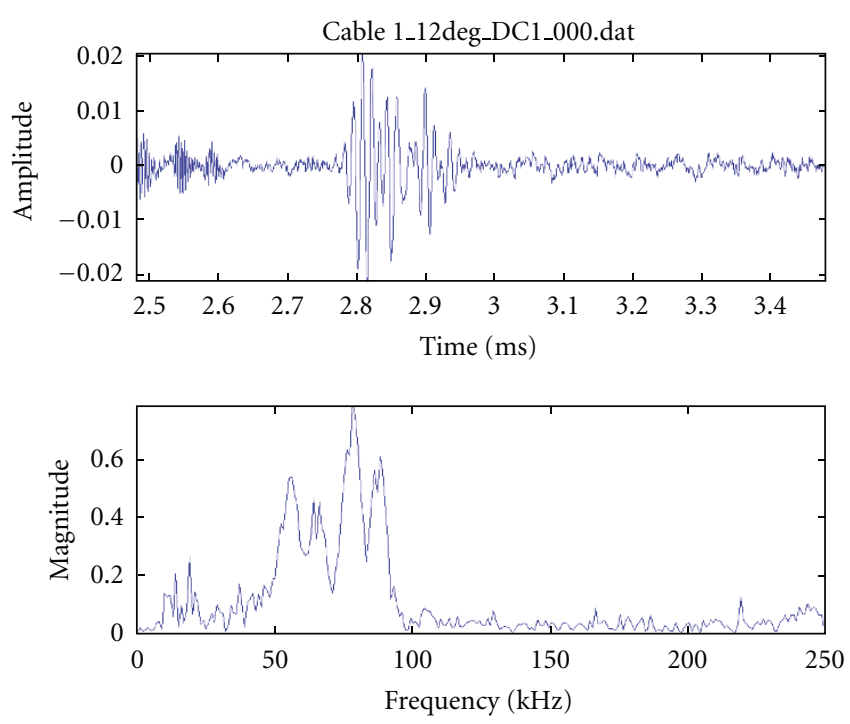

(c)

FIGURE 16: Signals and spectra for type C cable using test pulse DC1: (a) $3^{\circ}$ aspect response similar to broadside; (b) $8^{\circ}$ aspect return much reduced but still distinctive; (c) $12^{\circ}$ aspect, detection good but at lower SNR reverberation affects spectral signature.

In each layer $n$, the wave equation can be written as:

$$
\vec{u}^{n}=-\vec{\nabla} \psi^{n}+\vec{\nabla} \times \vec{A}^{n},
$$

where $\psi^{n}$ and $\overrightarrow{A^{n}}$ are given by

$$
\begin{aligned}
& \psi^{n}=P_{0} \sum_{m=0}^{+\infty} i^{m} \epsilon_{m}\left[c_{m}^{n} J_{m}\left(k_{l}^{n} r\right)+d_{m}^{n} N_{m}\left(k_{l}^{n} r\right)\right] \cos m \theta, \\
& \overrightarrow{A^{n}}=P_{0} \sum_{m=0}^{+\infty} i^{m} \epsilon_{m}\left[e_{m}^{n} J_{m}\left(k_{l}^{n} r\right)+f_{m}^{n} N_{m}\left(k_{l}^{n} r\right)\right] \sin m \theta
\end{aligned}
$$

where $N_{m}$ is a Bessel function of the second kind.

There are three boundary conditions for the watercylinder interface: (i) the normal component of displacement is continuous,

(ii) the tangential component of shearing stress is null,

(iii) the pressure in the fluid equals the normal component of stress in the solid.

There are similarly three boundary conditions for each interface inside the cylinder:

(i) the normal component of displacement is continuous,

(ii) the tangential component of shearing stress is continuous,

(iii) the normal component of stress is continuous. 


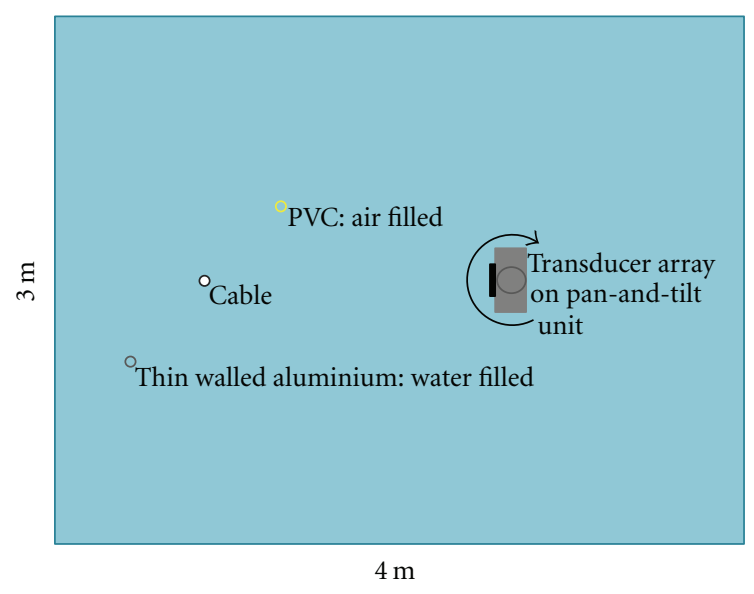

FIgURE 17: Test tank setup (plan view) with multiple narrow cylindrical targets. Transducer array is panned across the targets to get responses over the whole beamwidth.

The system of equations resulting from these limiting conditions allows us to solve the coefficients $b_{m}, c_{m}^{n}, d_{m}^{n}$, $e_{m}^{n}$, and $f_{m}^{n}$. The $b_{m}$ coefficients permit computation of the backscattered echo. Figure 14 shows a match between the theoretical and empirical results for the $32 \mathrm{~mm}$ diameter cable. The spacings between the strong notches are well represented. The notch positions are sensitive to relative phases of contributing echoes and are readily affected by any imprecision in values for acoustic properties of the materials. The match would be expected to improve with incorporation into the model of more accurate values for these parameters.

Designed to mimic the natural sonar of the bottlenose dolphin, the bioinspired wideband sonar has an excellent response over the frequency band $30-130 \mathrm{kHz}$. According to the resonance scattering theory (RST) [20], a resonant object offers strong spectral oscillations at $k a$ values between 2 and 50 , where $k=2 \pi / \lambda$ represents the wavenumber, and $a$ represents some key target dimension, for example, diameter. In the case of the cables available for test, $k a$ values are included in the range [2:40] so this bandwidth is expected to offer the characteristic oscillations needed for recognition.

\section{Cable Responses}

Covering all experiments, around 10000 target echo measurements have been gathered. Responses have been measured for a range of cable aspects with good, consistent results seen to better than $\pm 15^{\circ}$. Beyond $\pm 10^{\circ}$ the higher frequencies are lost progressively due to the frequencydependent nature of the transducer beamwidths. Data have been gathered mid-water for the first three cable types and for other thin cylindrical objects to test discrimination capability. Further experiments have been directed to detection of cables buried in soft sand sediment. A number of different wideband pulse types have been used in each experiment.

5.1. Free Water Response: Cable Type C. Figure 15 shows two measured responses for a cable using two different bioinspired wideband pulses. The first spectrum is measured for the cable in free water, the other with the cable lying against the concrete tank floor. The red arrows indicate predicted positions for spectral notches derived from the simple cable model. A more complex model and improved knowledge of the acoustic properties of the cable materials will be required to generate a higher fidelity model to describe more of the spectral variation. Note that in this figure raw spectra are presented and no account has been taken of the influence of the pulse shape.

5.2. MultiAspect Responses. In Figure 16, several responses are shown from the mid-water configuration given in Figure 2 to indicate how returns degrade away from broadside aspect. Under tank conditions using a single transmit/receive pair, the characteristic cable response is maintained to around $\pm 10^{\circ}$. At greater angles a response is still seen well above the noise floor, but it is corrupted by reverberation. Figure 16(c) represents the limit of the experimentation possible within the confines of the test tank. Space constraints also limit the length of cable that can be tested and restrict the degree of rotation possible. Under less constrained, deeper water conditions we can expect the recognisable response to be maintained beyond $\pm 12^{\circ}$ and matched receivers with an angular offset will increase the range over which a characteristic response can be measured still further.

5.3. Target Discrimination. Since the intended use of the wideband response is for classification, further tests with a small number of alternative narrow cylindrical targets have been undertaken. Given the limited tank space available these experiments were conducted with the cables and other cylinders suspended vertically in the water and with the transducers rotated accordingly, see Figure 17.

Figure 18 gives responses for the three targets to pulses DC1 and DC3. Whilst the former gives an excellent SNR for detection, there is little discriminatory potential between the responses for the cable and the strong specular response of the air-filled PVC tube. Signal DC3 provides more energy around the notch seen in the cable response at $\approx 65 \mathrm{kHz}$, so the resulting notch in the echo spectrum using DC3 becomes a useful discriminatory feature. A good match to the rest of the cable response provides a relatively high level for detection. Note that these targets are of very similar dimensions and have similar target strengths. It is highly unlikely they could be distinguished using a conventional imaging sonar.

5.3.1. Clutter Objects. Discrimination from clutter objects poses a less severe test, though multiaspect responses must be taken into account. For example, responses for a dense concrete block are shown in Figure 19. In this case the largest flat face of the block is facing the transducer and relatively consistent echoes are seen, reflecting the composition of the transmit signal.

The key determinants for the block response when it is rotated are the principal diffraction points defined by the 


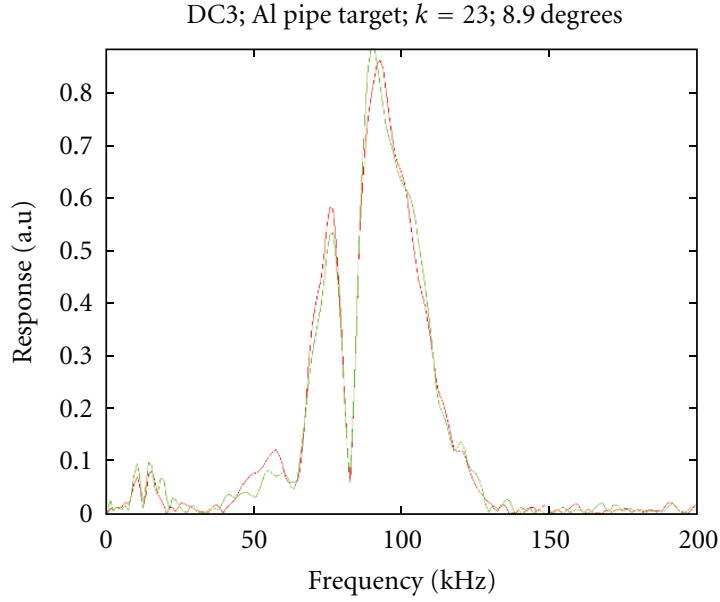

(a)

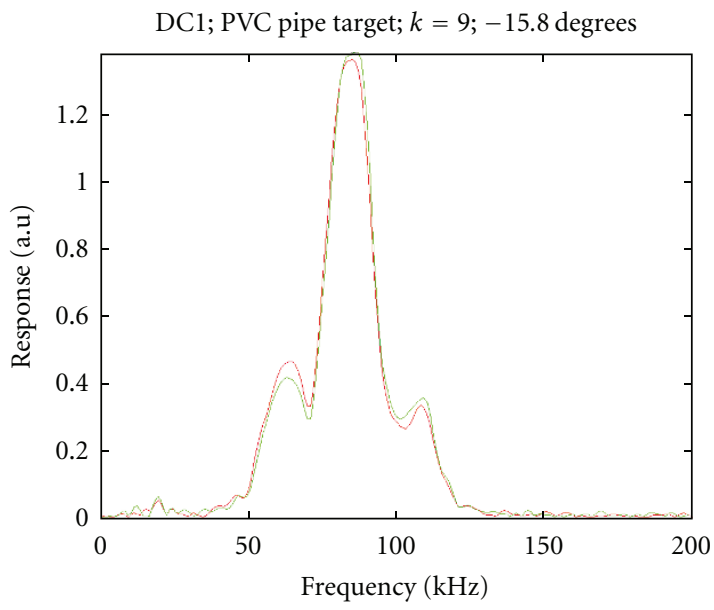

(c)

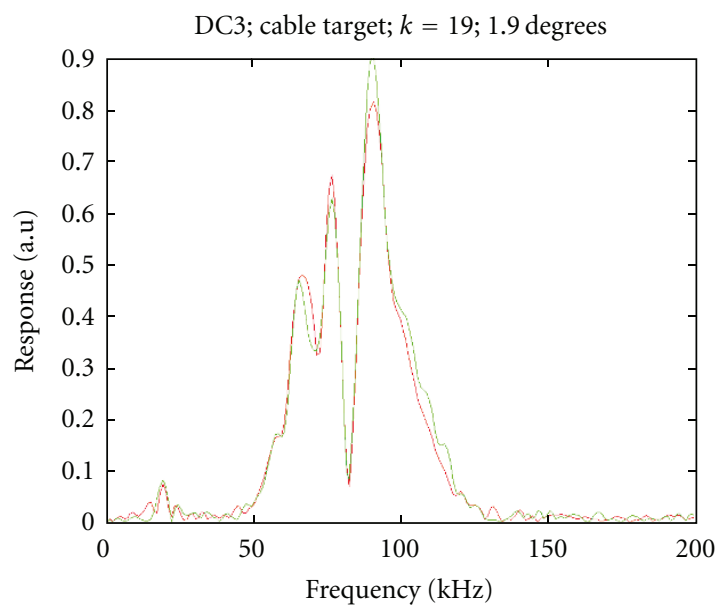

_ Left channel

__ Right channel

(e)

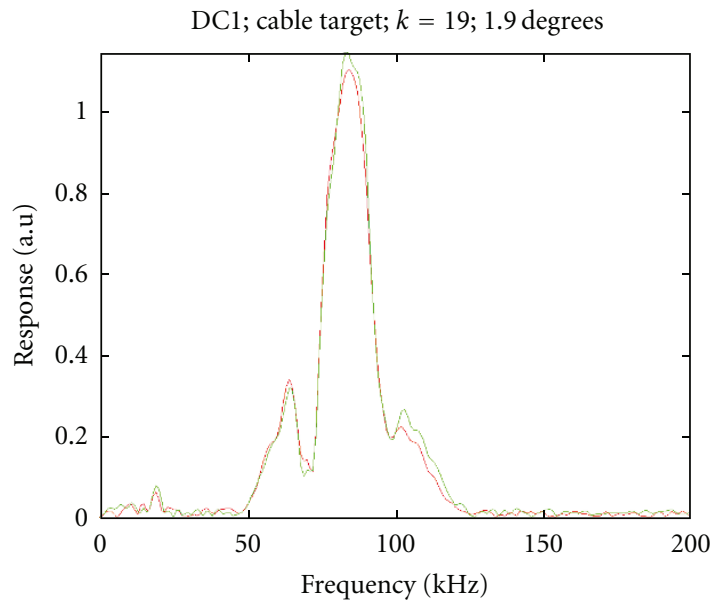

(b)

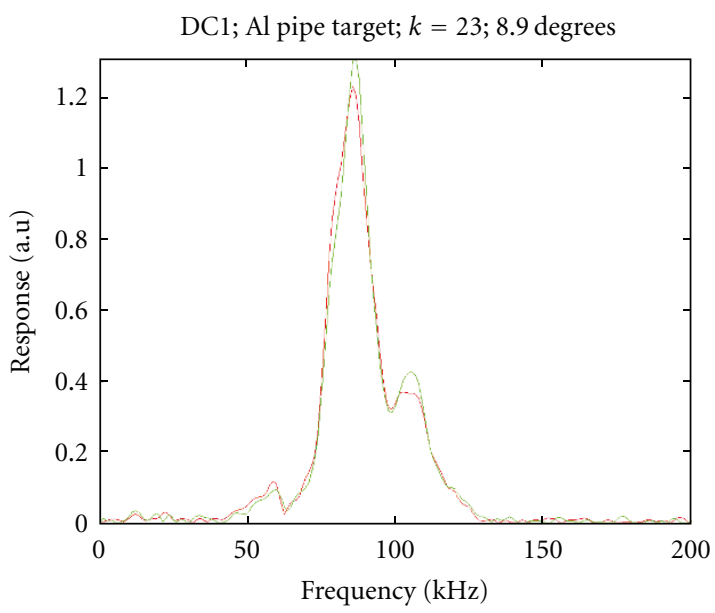

(d)

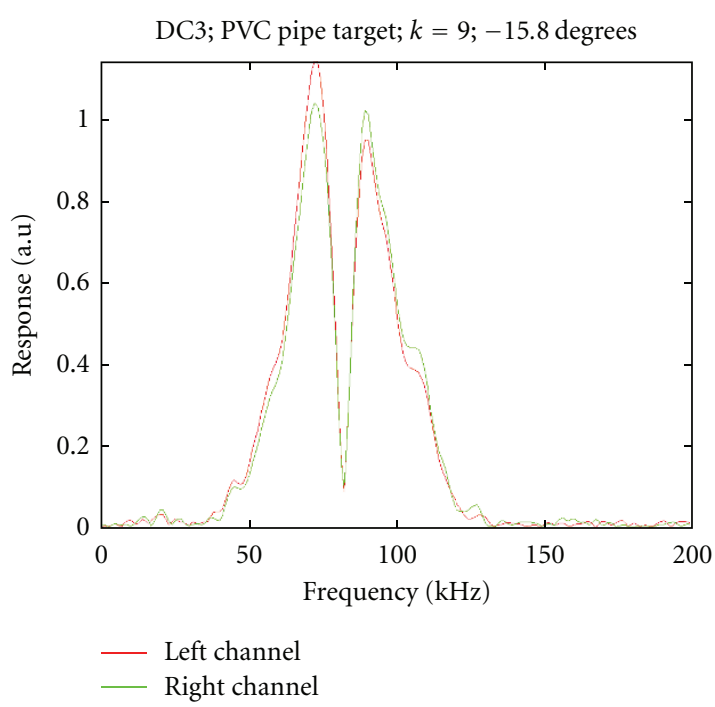

(f)

Figure 18: Target echo spectra using pulses DC1 and DC3 for a water-filled aluminium tube, a type C cable and an air-filled PVC tube. 


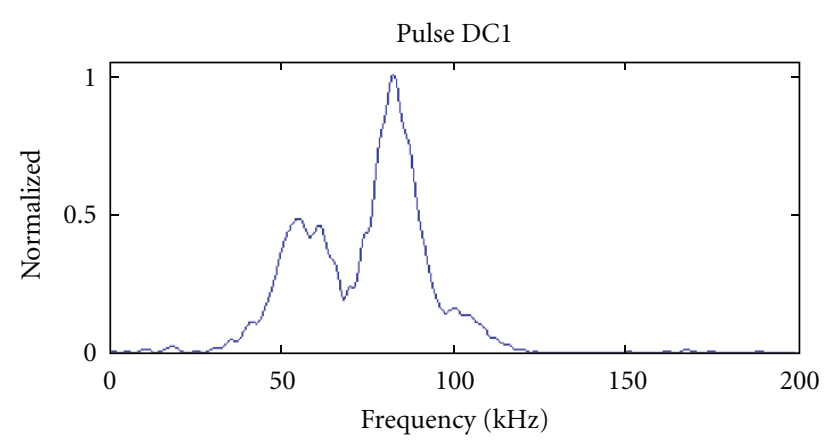

(a)

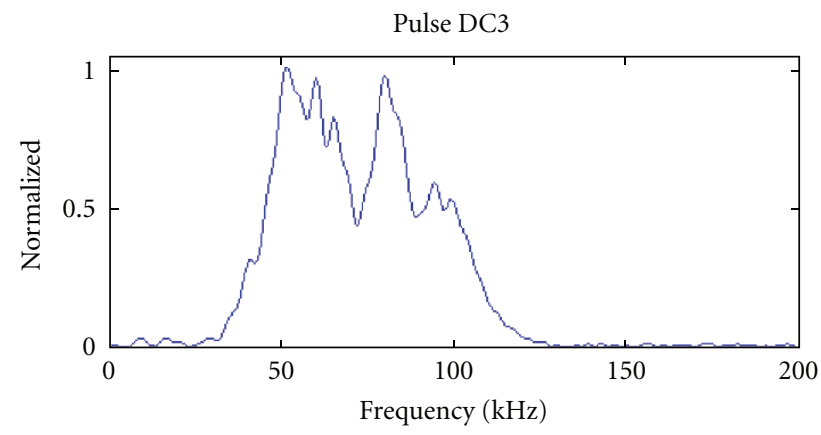

(c)

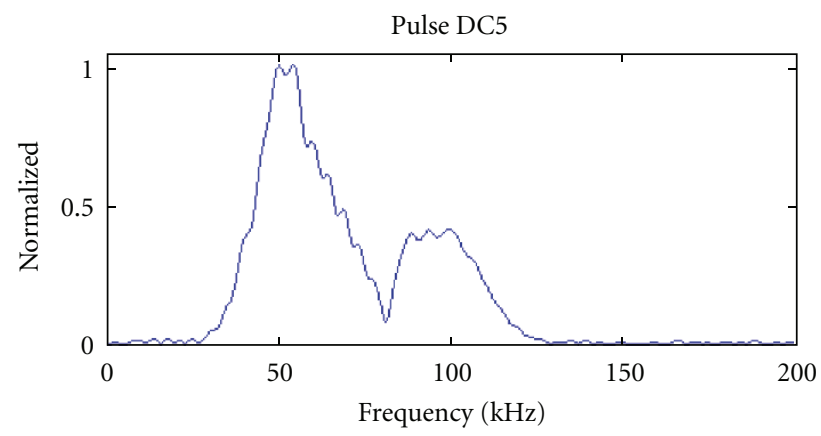

(e)

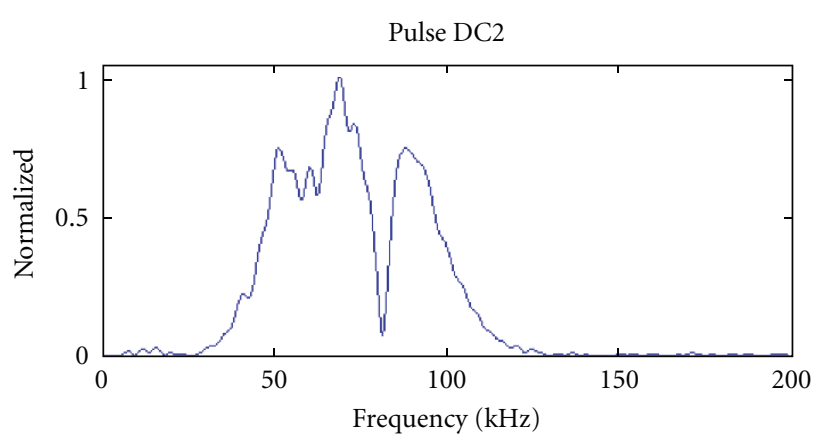

(b)

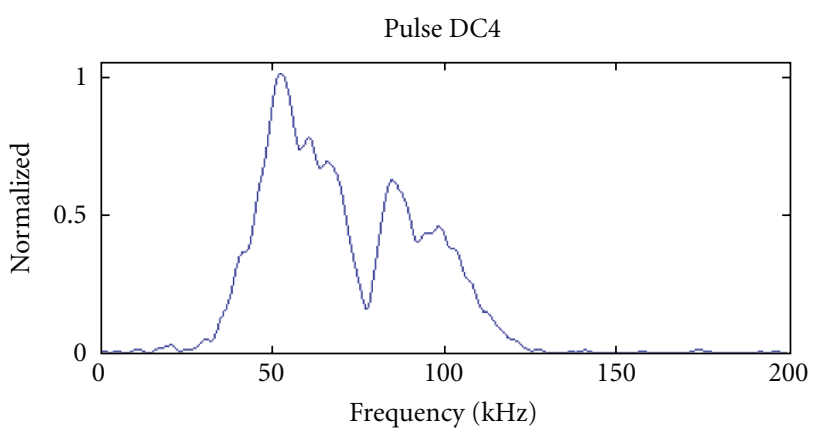

(d)

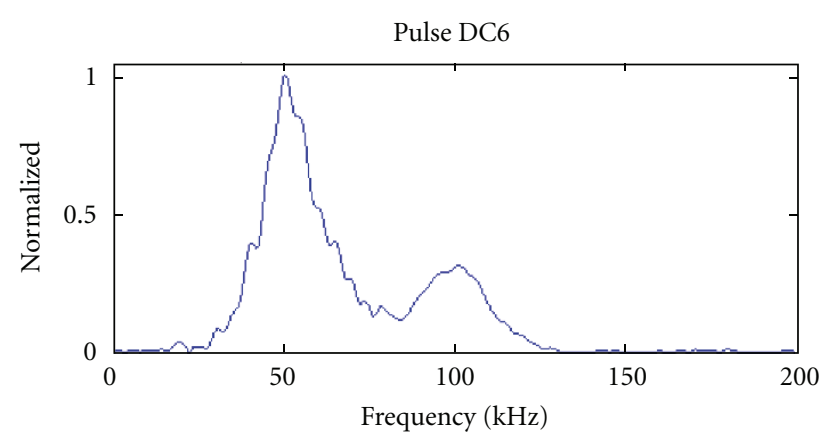

(f)

FiguRE 19: Spectral responses for a large concrete block, pulses DC1-6.

largest scatterers associated with the target. In this case a diffuse scattering from the rough surface is seen along with major backscattering points at the corners of the block. The spacing of these scatterers gives returns, which are more widely spaced in time and correspond to periodic oscillations in the echo spectra, see Figure 20. Note that the general shapes of the spectra are similar to those for the flat face returns. These responses are very different to the cable and pipe responses and can be readily distinguished. In other projects, we have used wideband responses to classify and identify a variety of objects, including cylinders, cones, rocks, bricks and concrete blocks $[8,13]$. All of these could be considered as clutter in the current context. For each object, there is typically a characteristic aspect, which provides excellent discrimination. In the absence of these characteristic responses, discrimination is still possible from multiaspect data.
5.4. Differential Responses between Cable Types. In this experiment, the cables are positioned at $1.5 \mathrm{~m}$ range from the sonar, mid-water at a depth of $0.8 \mathrm{~m}$. The four cable types, A, $\mathrm{B}, \mathrm{C}$, and D have been tested. Responses are given for the range of double chirp pulses to indicate that discrimination will be achieved more readily using some pulses than others. In each case the responses have been normalized, since it is the shape of the response we are most interested in. All responses are measured at broadside aspect.

Figure 21 gives the responses for cable type A, the $21 \mathrm{~mm}$ diameter white plastic sheathed cable. The variation seen between pulse types is in part due to the spectral shape of the pulse and in part due to the echo spectral response. Figure 22 shows the responses for the type B, narrow black cable. Here contributions from the ring of strengthening cables surrounding the conducting core give rise to typically more oscillatory responses. 


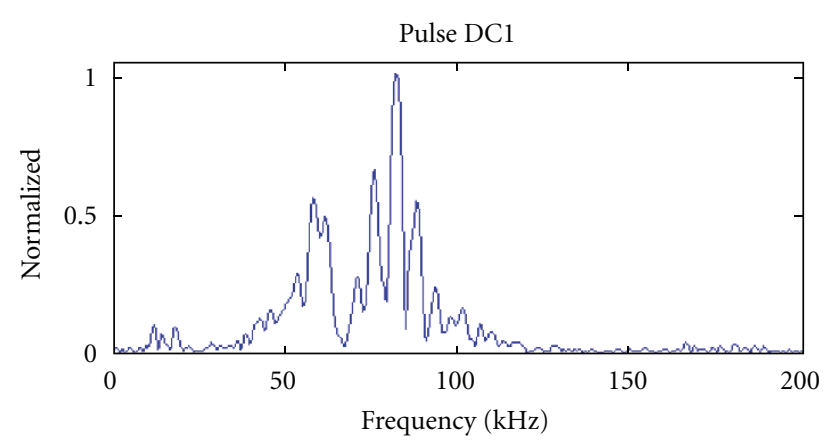

(a)

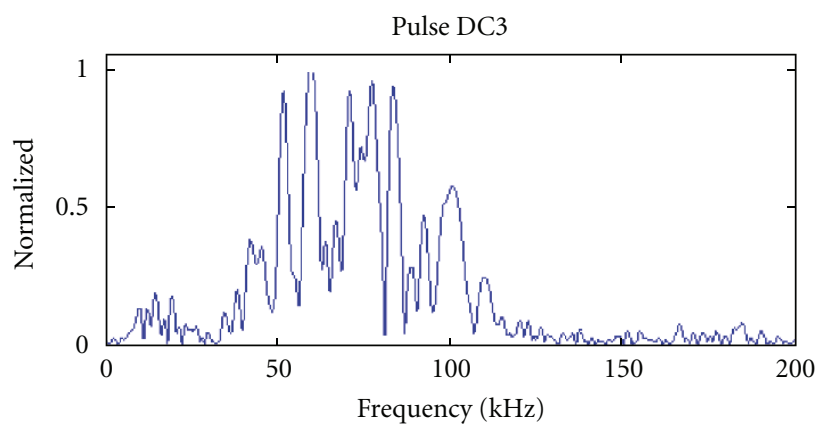

(c)

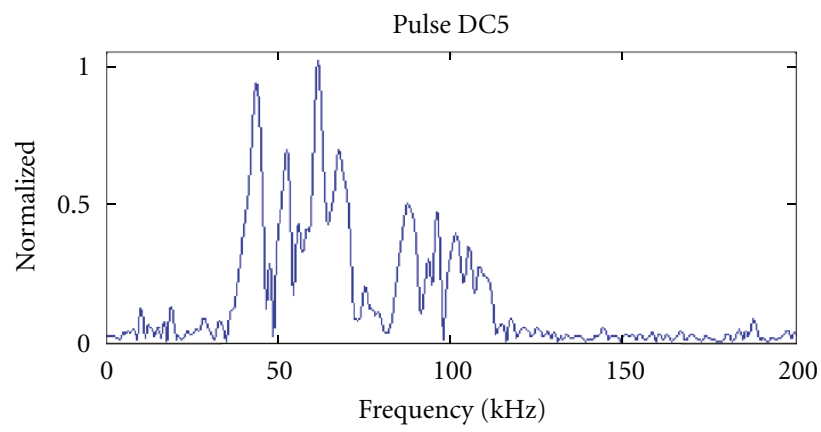

(e)

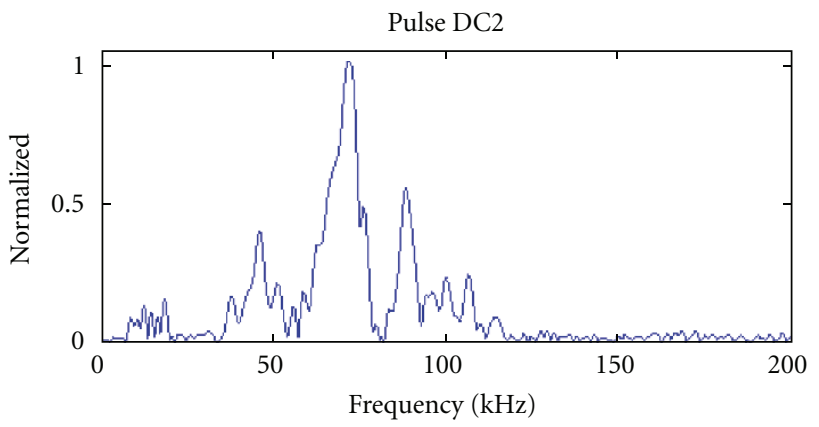

(b)

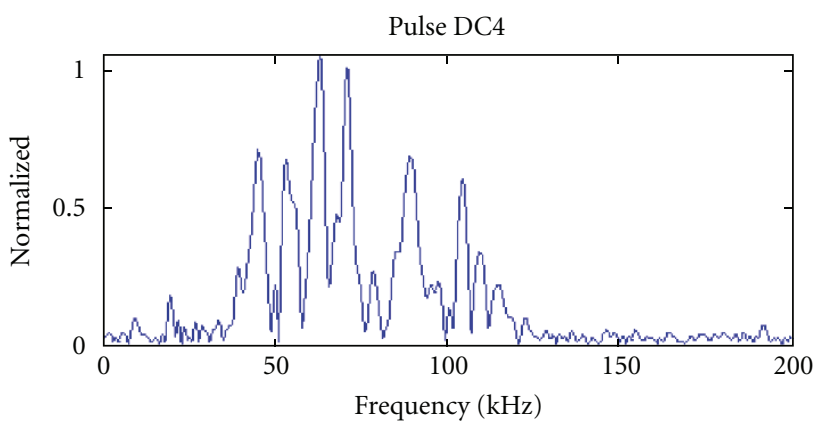

(d)

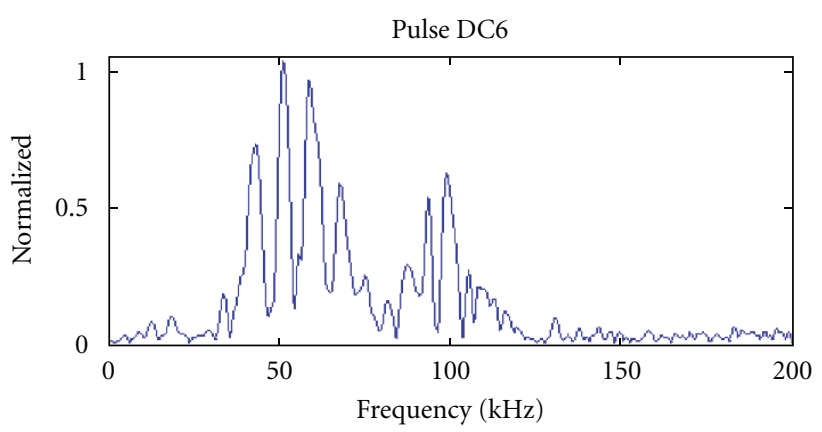

(f)

FIGURE 20: Spectral responses for the same concrete block tilted relative to the sonar beam axis $\left(\approx 30^{\circ}\right)$, pulses DC1-6.

In Figure 23, the responses to DC1 and DC2 for the larger $32 \mathrm{~mm}$ diameter type $\mathrm{C}$ cable are similar to those for the type A cable. With more separation between the constituent chirps, using signals DC3-DC6, the responses are more varied. DC5 provides a good candidate for discrimination given the strong oscillations in the $50-80 \mathrm{kHz}$ band. The similarities seen in the echo spectra between types $\mathrm{A}$ and $\mathrm{C}$ reflect the similarities in these cables physical structures.

The responses for the SL17L cable D are shown in Figure 24. This cable presents another distinctive set of responses to the six DC pulses. In mid-water consistency between pings is very good, but this cable response does have the lowest SNR as indicated by the relatively higher background noise levels. These results give confidence that all of these cable types can be discriminated using the wideband approach.

\section{Buried Cables and Sediment}

The tank configuration used for the buried cable tests is shown in Figure 25. Type $\mathrm{C}$ cable responses have been tested at broadside incidence only. Five sections of cable were used, each $3 \mathrm{~m}$ in length. One was placed on the surface of the sand, one was buried immediately subsurface and a further three sections were buried at depths of $20 \mathrm{~mm}, 50 \mathrm{~mm}$ and $100 \mathrm{~mm}$, to the top of the cable. The cables were horizontally spaced at $200 \mathrm{~mm}$. The sediment layer used for these tests is characterised as very fine sand according to APL-UW definitions, with a variable grain size of between 0.2 and $2 \mathrm{~mm}[21]$.

Responses were seen for cables at all burial depths, being more pronounced at steeper grazing angles (i.e., closer to normal incidence). The greatest responses, corresponding to maximum penetration of acoustic energy into the sediment 


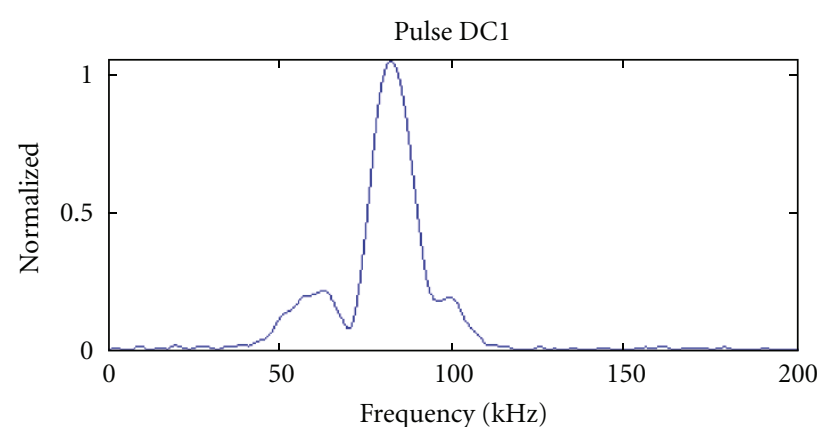

(a)

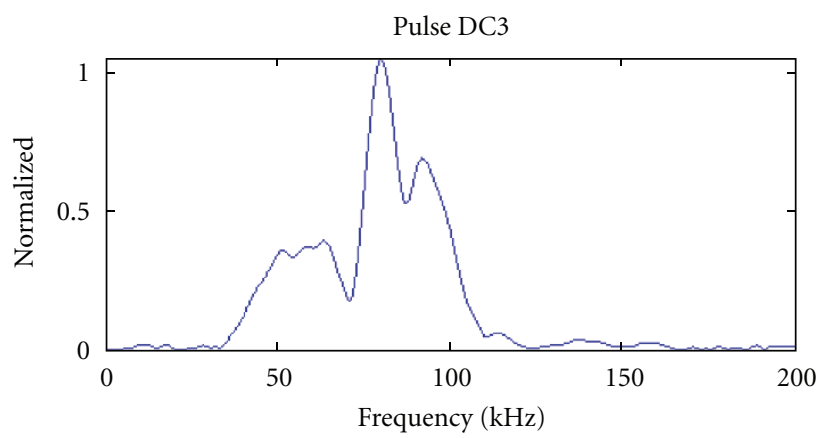

(c)

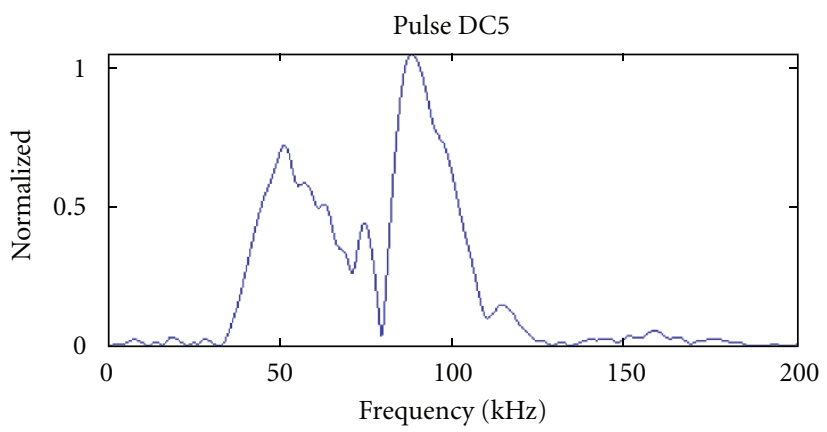

(e)

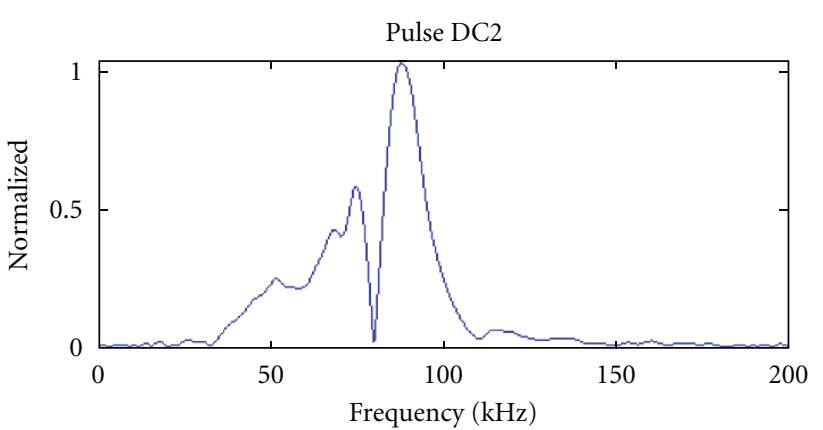

(b)

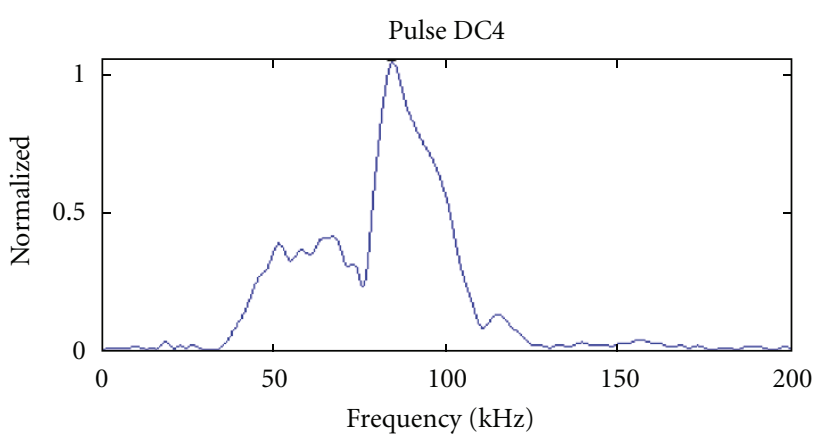

(d)

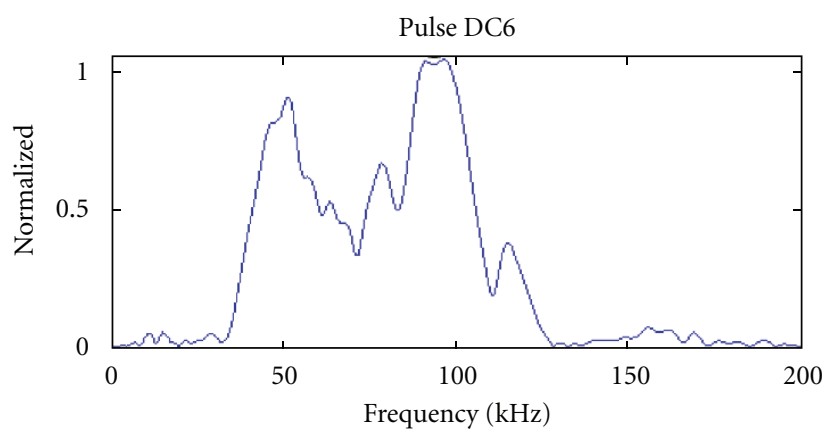

(f)

FIgURE 21: Spectral responses for cable type A, pulses DC1-6.

layer, were seen at a sonar depression angle of around $50^{\circ}$. Figure 26(a) collates data from some 30 pings with the sonar head tracking from the shallowest to the deepest cables in $10 \mathrm{~cm}$ increments. The vertical axis is range. The horizontal axis is ping number. Figure 26(b) shows the expected responses given the sensor beamwidth and assuming no losses. In Figure 26(c) the two are overlaid to aid interpretation. The graphic shows that all of the cables are detected, but that the deeper cable responses are seen only for steeper grazing angles.

As described in Section 4, the major contributions to the cable echo structure come from the specular return and the surface acoustic waves travelling in the outer copper conducting layer. In the idealised case, depicted in Figure 27, the relative path lengths are affected by refraction at the water/sediment boundary. Overall the difference comes down to a few millimetres extra propagation in sand rather than water for the surface wave returns, but this will still have a noticeable impact on the relative phases of the contributing echoes.

In practice, the situation is complicated by the fact that the burial depths at which we can detect the cables are likely to be less than the pulse length. In this situation the cable echo is inseparable from the sediment surface response. In Figure 5 we show normalized responses for the cable section lying on the sand surface and for the shallowest buried cable (top surface flush with the sediment) for a grazing angle of $27^{\circ}$. A Gaussian windowed FM pulse with a $6 \sigma$ nominal bandwidth of $10-150 \mathrm{kHz}$ was used.

Considerably more experimentation will be required to sort out phase changes in the cable responses with burial, but these brief experiments have demonstrated that detection of a buried cable is possible using the wideband sensors facilitating tracking when cables go into burial. Steeper grazing angles will be required, indicating that a mission profile might have to be modified to deal with cable burial. 


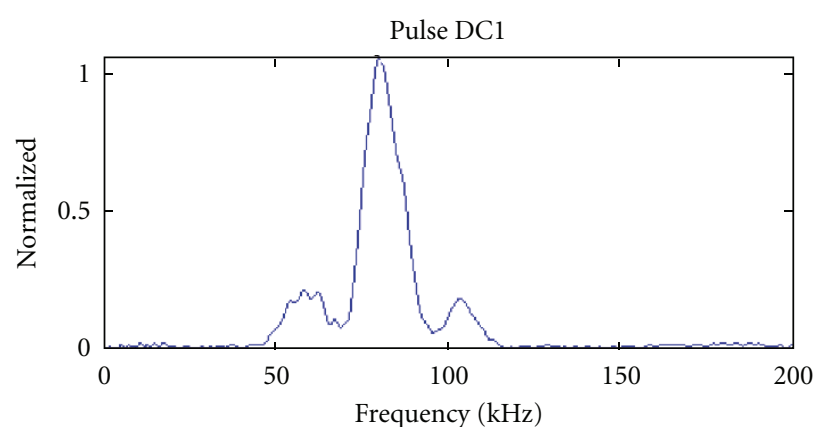

(a)

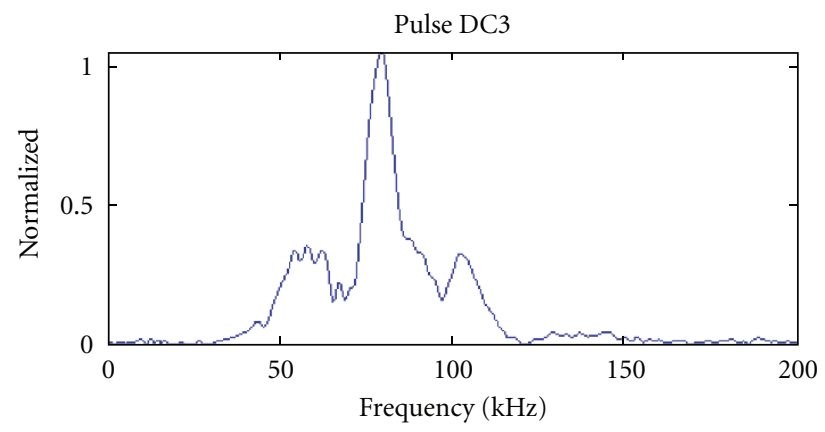

(c)

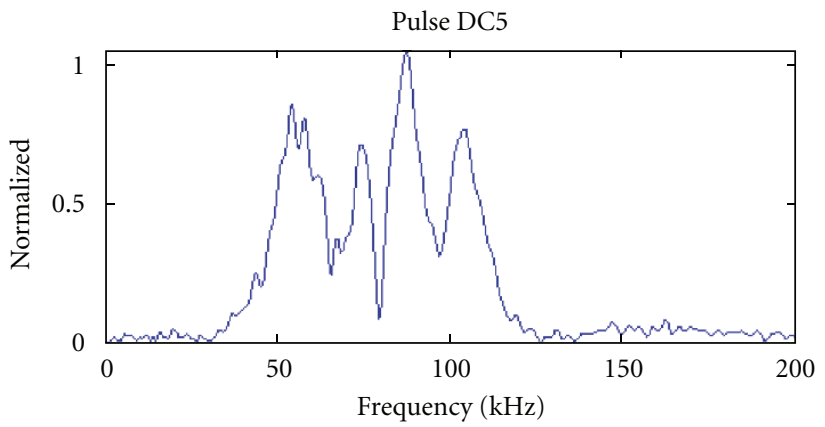

(e)

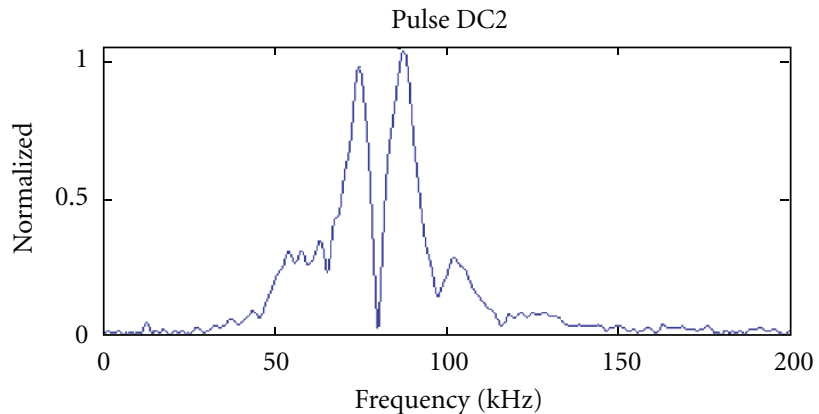

(b)

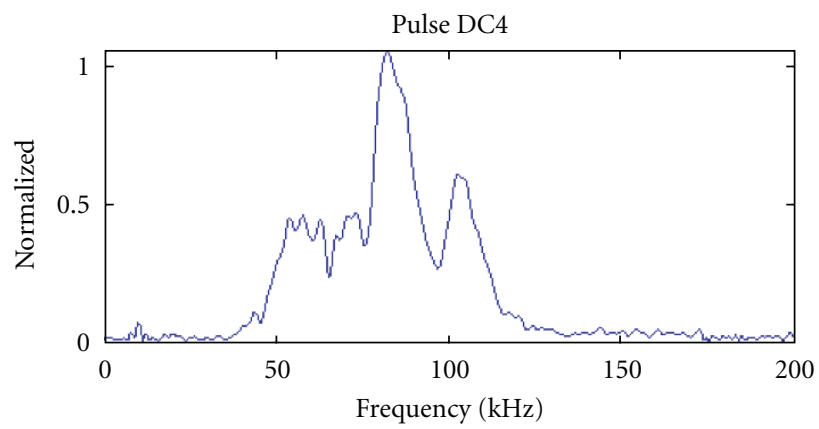

(d)

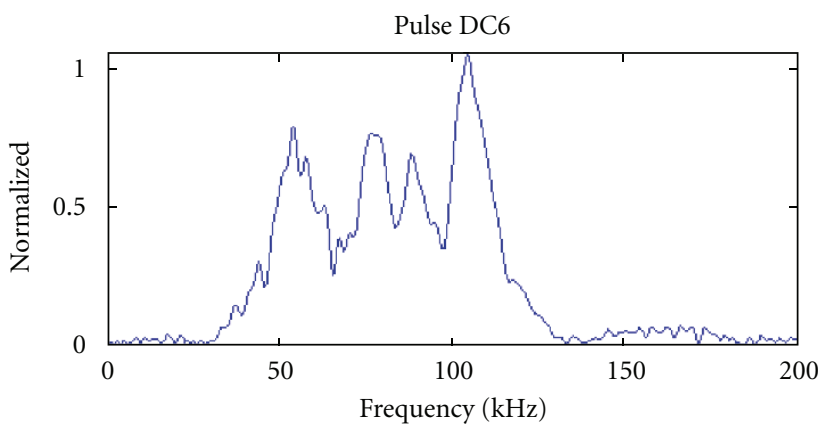

(f)

FIgURE 22: Spectral responses for cable type B, pulses DC1-6.

Robust identification from buried cable returns will require a better understanding of the effect of burial on the cables spectral signatures.

6.1. Reverberation Limits on Coarse Sediment. The coarse sediment used for the experiments is a mixture of coarse sand and grit, with particle sizes varying from around $0.2 \mathrm{~mm}$ to more than $4 \mathrm{~mm}$. This presents more difficulty for detection because the reverberation noise level is directly related to the bottom scattering strength:

$$
\mathrm{RL}=\mathrm{SL}-2 \mathrm{TL}+S_{v}+10 \log _{10} \frac{c \tau}{2} \psi r^{2},
$$

where SL is the source level, TL is the transmission loss, $\tau$ is the pulse duration and $\psi$ is the solid angle derived from the ideal beam pattern. Figure 6 displays the bottom scattering strength for three different sediment types (very fine sand, sandy gravel and rough rock) relative to grazing angle. This figure illustrates two important points for the detection process. Firstly, the rougher the sediment, the higher the reverberation level. Secondly, the grazing angle plays an important role in determining the reverberation noise level. For rough sediments, a lower grazing angle will provide better results. Over the very coarse sand and in these experiments low grazing angles for the sonar are preferred to improve SNR.

6.1.1. Experimental Results. Figure 7 shows a series of responses for the cable at two different grazing angles, $45^{\circ}$ and $27^{\circ}$. In these experiments the sensor is tracked along the tank and passes over the cable. In the images each horizontal line gives the amplitude values for a single sonar ping. The sensor position is represented on the vertical axis, with range to target echoes in the horizontal axis. The constant vertical line around $0 \mathrm{~m}$ range is the direct coupled response. There are three major echo responses following diagonal lines 


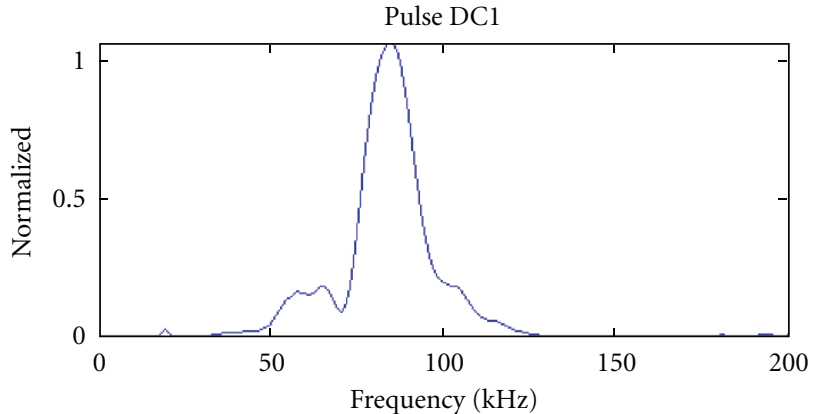

(a)

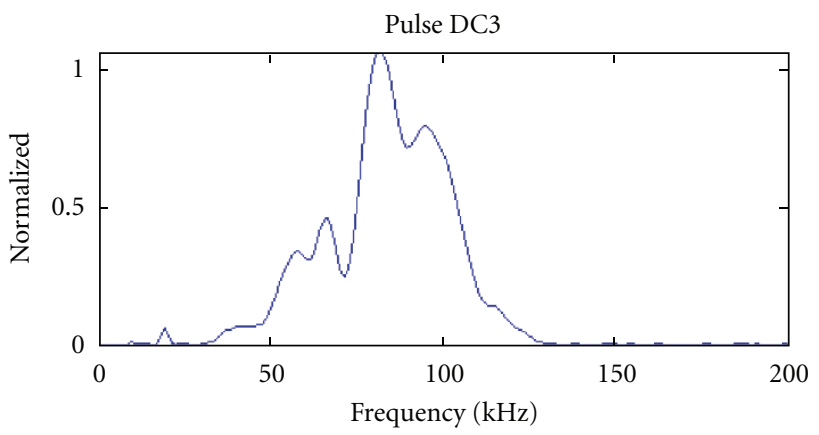

(c)

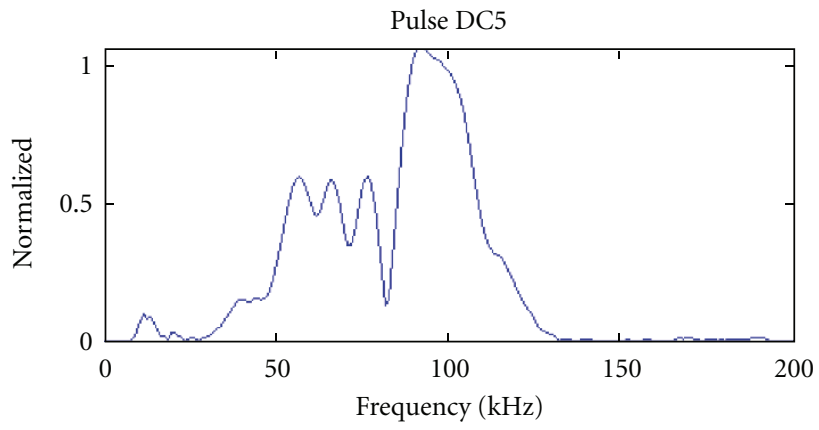

(e)

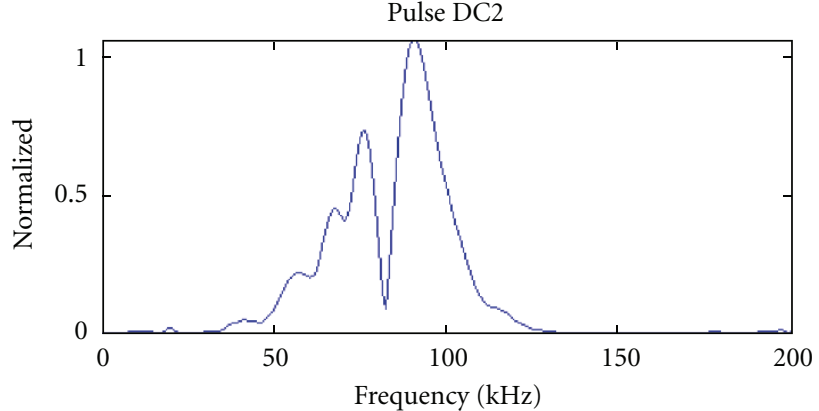

(b)

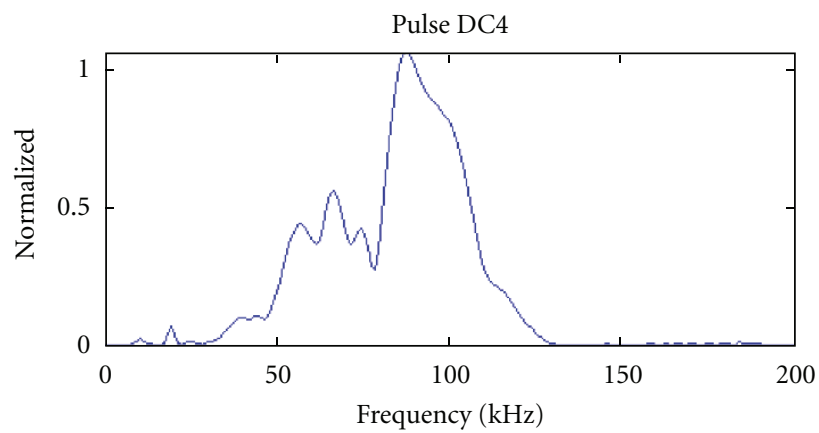

(d)

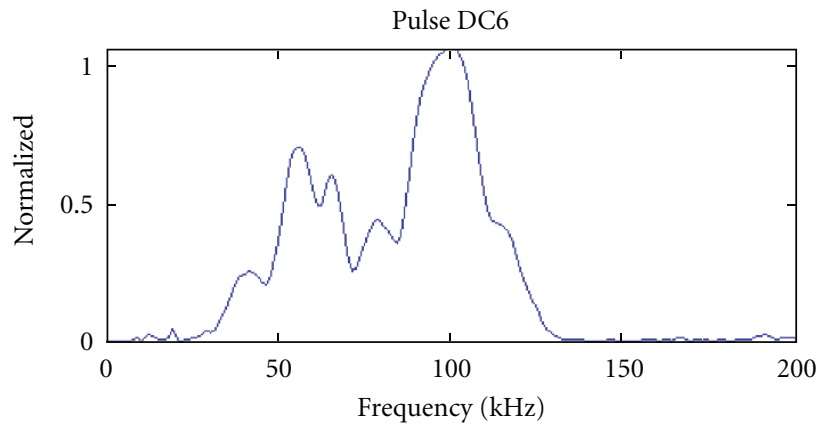

(f)

FIGURE 23: Spectral responses for cable type C, pulses DC1-6.

across the image as the sensor is moved ping to ping. These correspond to a separating board on the tank floor, then the cable and finally a large response from the tank wall. In contrast to previous experiments with the fine sand, the cable is hard to discern at the steeper grazing angles. It stands out well at the shallower angle.

To illustrate these points further spectral responses are provided as in the free water experiments above for the SL17L cable D. At the $45^{\circ}$ grazing angle the cable response is completely buried in reverberation noise at the beam centre presenting an extremely difficult task for detection and identification. In fact, better results are obtained by making use of the wide beam pattern and looking at the periphery of the beam, in effect using a shallower grazing angle. This effect is in part due to the frequency dependant beamwidth. Further work should be done on other pulse types to look at what will be the best for different scenarios of operation. Figure 8 shows such a response to a DC4 pulse with the cable echo arrowed.
Figure 9 presents the spectra for each of the six double chirp pulses. Though SNR is relatively low and the target response is well below the main lobe reverberation, the spectra do follow the general pattern of the responses measured mid-water. Improved post-detection filtering and noise suppression would be of benefit in classifying these returns.

At the $27^{\circ}$ grazing angle we can use the main lobe returns without losing the cable echo in reverberation noise. Figure 10 gives spectra for the shallower grazing angle and the match with the mid-water measurements is better. Finally we illustrate the variability in response over the coarse sediment by plotting a sequence of 43 DC 3 echoes recorded at $5 \mathrm{~cm}$ intervals along a $2 \mathrm{~m}$ cable segment. In addition to the sediment reverberation, these data are compromised by cable curvature, tank wall returns, disturbances in the sediment surface and ambient noise sources. Figure 11 is the record of the echo magnitudes. Figure 12 presents a waterfall plot of the cable echo spectra. 


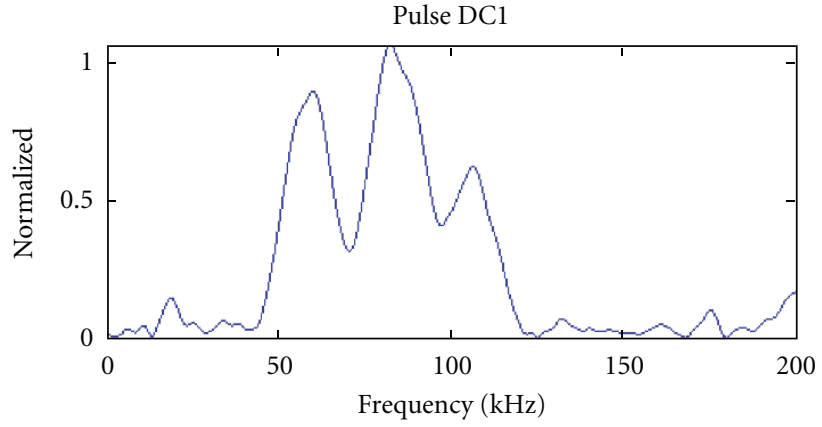

(a)

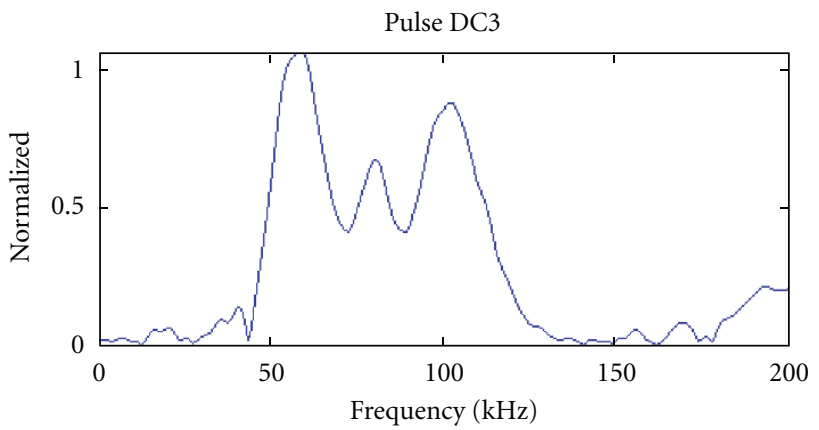

(c)

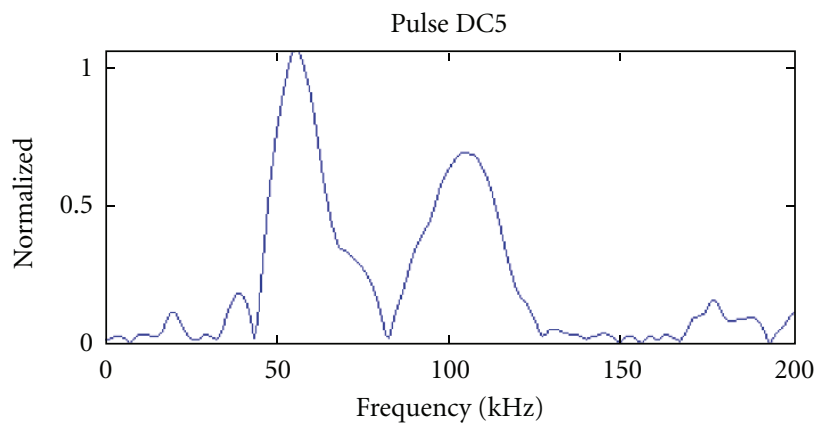

(e)

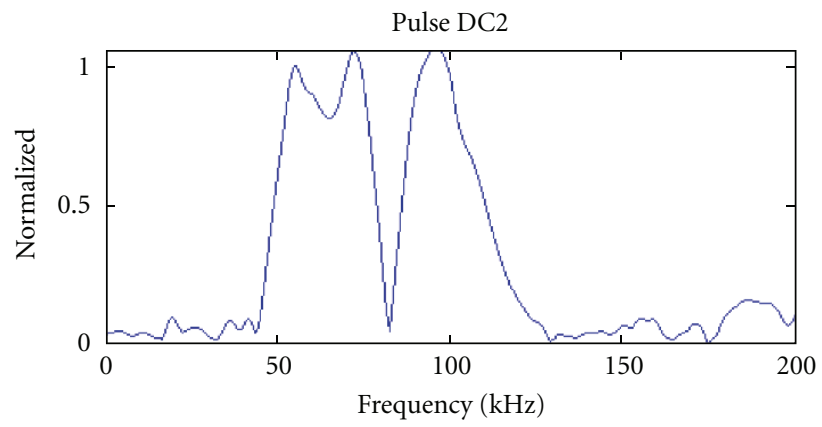

(b)

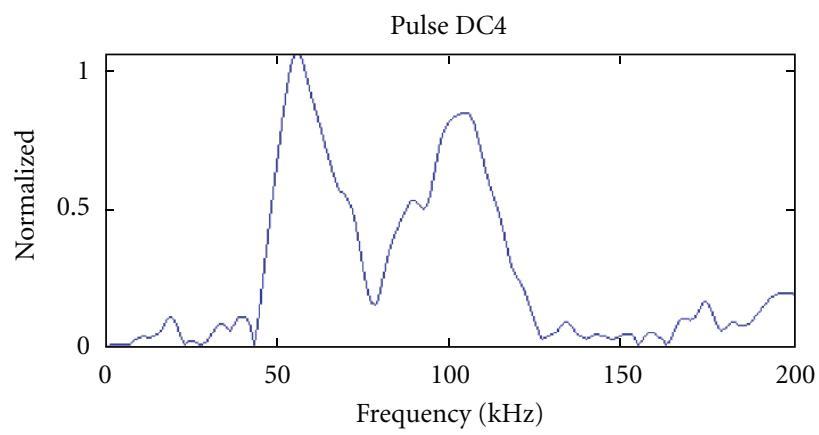

(d)

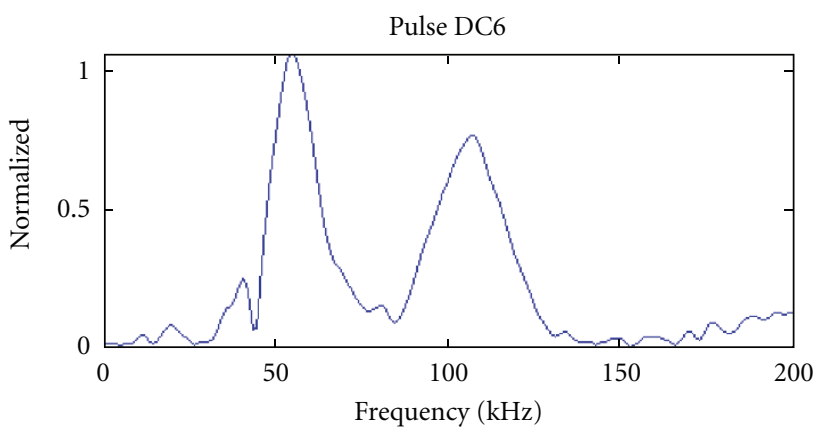

(f)

FIgURe 24: Spectral responses for bare SL17L cable D, pulses DC1-6.

\section{Conclusions}

The data gathered during these experiments have indicated good cable detection and identification potential for the bioinspired wideband sensors. Consistent characteristic responses have been measured for each of the cables tested and, given our experience with wideband responses for a wide variety of targets, these seem well suited to discrimination from seabed clutter objects. Usable ground coverage is available with the existing sensors and can be maximised by using a stereo pair of receivers. The SL17L cable D does provide a significantly reduced SNR and we have suggested that shallower grazing angles will be required for the smaller cable types, particularly over more reverberant surfaces. The type B cable presents greater difficulties through a more variable response due to its more complex structure. In all of these cases the detection will be improved by integrating over a sequence of cable echoes. This adds some complexity

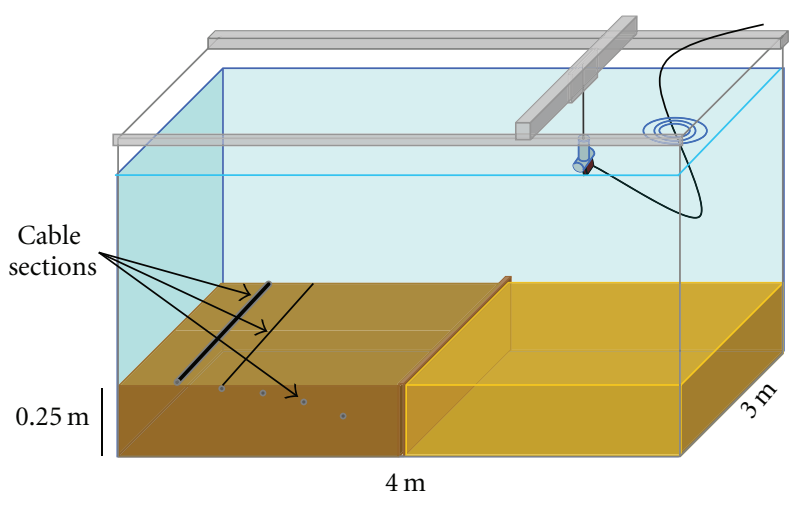

Figure 25: Test tank setup for buried cable responses.

to the cable detector, but will provide more robust detections to pass to the tracking module. 


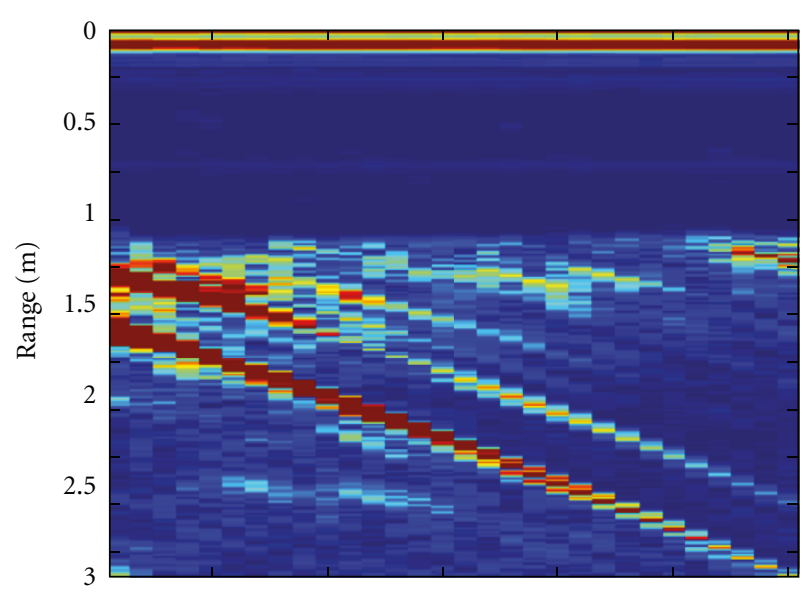

(a)

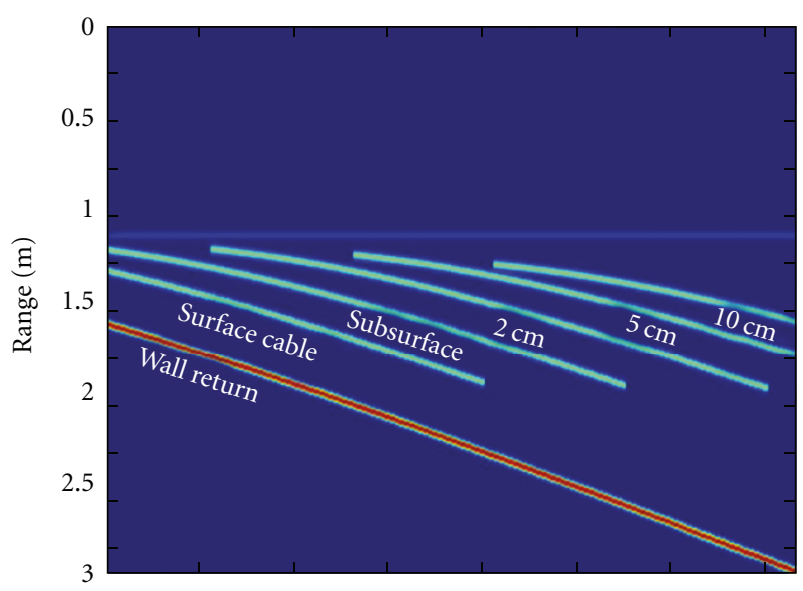

(b)

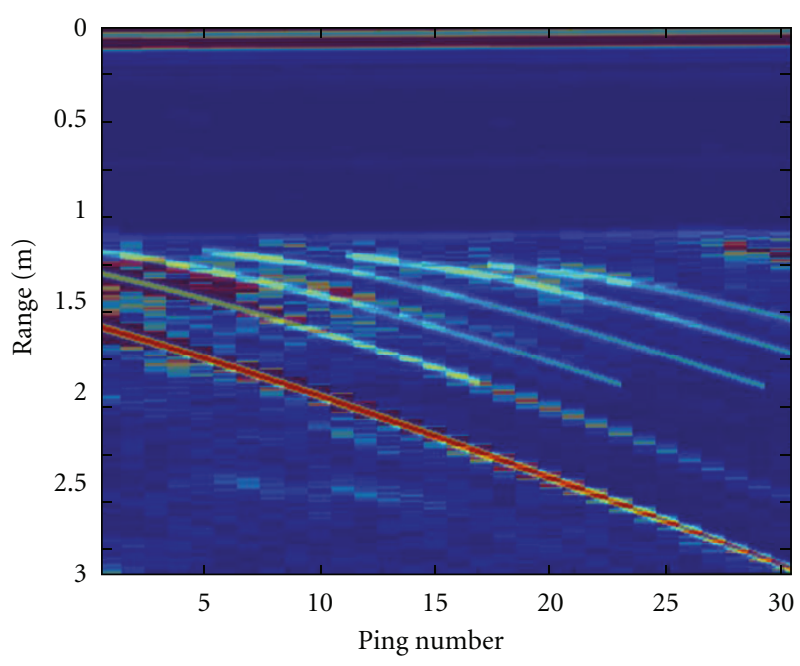

(c)

FIGURE 26: Graphic showing buried cable responses with angle of $27^{\circ}$ : (a) measured responses; (b) expected responses given sensor positions and beam patterns; (c) expected overlaid on measured responses.

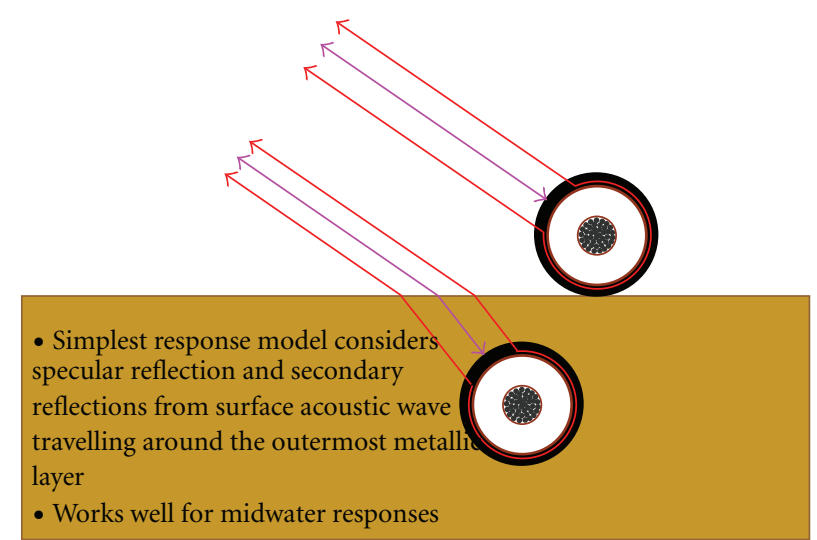

$$
\begin{aligned}
& \longleftrightarrow \text { Specular echo } \\
& \longleftrightarrow \text { Surface acoustic wave (outer Cu conductor) }
\end{aligned}
$$

Figure 27: Major contributions to echo structure come from the specular return and the surface waves travelling in the outer copper conducting layer. The relative path lengths are affected by refraction at the water/sediment boundary.

The key findings of this work are that the cables give a consistent response to the bioinspired signals wideband pulse. There has been strong agreement between the theoretical responses and the experimental results. Different cable types can be distinguished by their response and cables can be differentiated from other objects of similar scale/shape. When dealing with highly reverberant surfaces, shallower grazing angles are required to maintain good SNR. The wide beamwidth of the bioinspired wideband signal ensures good detection over a wide range of aspects $\left( \pm 20^{\circ}\right)$. Detection of buried cable is possible to depth of $\approx 10 \mathrm{~cm}$ in soft sediment using these types of signals. It was found that a $50-60^{\circ}$ grazing angle is optimal for detection in fine sand sediment. The return signal is modified due to path differences between returns in sediment layer, but still contains enough information to classify the cable types. The detection of buried cable is reverberation limited for coarser sediment types. Overall the results are good and indicate that the bioinspired wideband sensors can be used flexibly. They provide complementary information to the other sensors and can give more robust identification through characteristic spectral signatures.

\section{Acknowledgments}

This paper was funded by the US Naval Facilities Engineering Command (NAVFAC) in conjunction with SeeByte Ltd, Edinburgh, UK (http://www.seebyte.com/), and Sound and Sea Technology Lynwood WA USA (http://www.soundandsea.com/). The authors would also like to acknowledge previous enabling support from the US Office of Naval Research, the Space and Naval Warfare Systems Command (SPAWAR) San Diego, and the Defence 
Science and Technology Laboratory (DSTL), an agent of the UK Ministry of Defence. They would also thank the reviewers for their comments that helped improve the paper.

\section{References}

[1] W. Au, The Sonar of Dolphins, Springer, London, UK, 1993.

[2] R. Busnel and J. Fish, Animal Sonar Systems, Plenum, New York, NY, USA, 1980.

[3] P. Nachtigall and P. Moore, Animal Sonar: Processes and Performance, Plenum, New York, NY, USA, 1988.

[4] R. Kastelein and J. Thomas, Sensory Abilities of Cetaceans: Laboratory and Field Evidence, Plenum, New York, NY, USA, 1990.

[5] P. Nachtigall, "Odontocete echolocation performance on object size, shape, and material," Animal Sonar Systems, pp. 71-95, 1980.

[6] C. Capus, K. Brown, and P. Moore, "Extending the click taxonomy for the bottlenosed dolphin (Tursiops truncatus)," in Proceedings of the Institute of Acoustics (Bioacoustics '04), vol. 26, 2004.

[7] C. Capus, Y. Pailhas, K. Brown, D. M. Lane, P. W. Moore, and D. Houser, "Bio-inspired wideband sonar signals based on observations of the bottlenose dolphin (Tursiops truncatus)," Journal of the Acoustical Society of America, vol. 121, no. 1, pp. 594-604, 2007.

[8] Y. Pailhas, C. Capus, K. Brown, and P. Moore, "Analysis and classification of broadband echoes using bio-inspired dolphin pulses," Journal of the Acoustical Society of America, vol. 127, no. 6, pp. 3809-3820, 2010

[9] J. C. Isaacs and R. Goroshin, "Automated cable detection in sonar imagery," in Proceedings of the IEEE International Conference on Systems, Man and Cybernetics, pp. 2745-2750, 2009.

[10] Y. Pailhas, C. Capus, and J. Bell, "Modelling for obstacle avoidance sonar design," Proceedings of the Institute of Acoustics, 2005.

[11] D. A. Heiweg, P. W. Moore, S. W. Martin, and L. A. Dankiewicz, "Using a binaural biomimetic array to identify bottom objects ensonified by echolocating dolphins," Bioinspiration and Biomimetics, vol. 1, no. 2, pp. 41-51, 2006.

[12] C. Capus and K. Brown, "Short-time fractional fourier methods for the time-frequency representation of chirp signals," Journal of the Acoustical Society of America, vol. 113, no. 6, pp. 3253-3263, 2003.

[13] C. Capus, Y. Pailhas, K. Brown, J. Evans, and D. Willins, "Underwater detection classification and tracking using wideband sonar," in Proceedings of the 3rd International Conference and Exhibition on Underwater Acoustic Measurements: Technologies \& Results, 2009.

[14] H. Lamb, "On waves in an elastic plate," Proceedings of the Royal Society of London, vol. 93, pp. 114-128, 1917.

[15] T. N. Grigsby and E. J. Tajchman, "Properties of lamb waves relevant to the ultrasonic inspection of thin plates," IRE Transactions on Ultrasonic Engineering, vol. 8, pp. 26-33, 1961.

[16] S. G. Kargl and P. L. Marston, "Observations and modeling of the backscattering of short tone bursts from spherical shell: lamb wave echoes, glory, and axial reverberation," Journal of the Acoustical Society of America, vol. 85, no. 3, pp. 1014-1028, 1989.

[17] P. L. Marston, "Gtd for backscattering from elastic spheres and cylinders in water, and coupling of surface elastic waves with the acoustic field," Journal of the Acoustical Society of America, vol. 83, pp. 25-37, 1988.

[18] R. Doolittle and H. Uberall, "Sound scattering by elastic cylindrical shells," Journal of the Acoustical Society of America, vol. 39, no. 2, pp. 272-275, 1966.

[19] R. Hickling, "Analysis of echoes from a solid elastic sphere in water," Journal of the Acoustical Society of America, vol. 34, pp. 1582-1592, 1962.

[20] G. C. Gaunaurd and H. Uberall, "RST analysis of monostatic and bistatic acoustic echoes from an elastic sphere," Journal of the Acoustical Society of America, vol. 73, no. 1, pp. 1-12, 1983.

[21] APL-UW, "High frequency ocean environmental acoustic models handbook," Tech. Rep. APL-UW TR 9407, University of Washington, Seattle, Wash, USA, 1994. 